\title{
Distributed Nash Equilibrium Seeking under Partial-Decision Information via the Alternating Direction Method of Multipliers
}

\author{
Farzad Salehisadaghiani ${ }^{\mathrm{a}}$, Wei Shi ${ }^{\mathrm{b}}$, Lacra Pavel $^{\mathrm{a}}$ \\ ${ }^{a}$ Department of Electrical and Computer Engineering, University of Toronto, Toronto, ON M5S 3G4, Canada \\ farzad.salehisadaghiani@mail.utoronto.ca, pavel@control.utoronto.ca. \\ ${ }^{\mathrm{b}}$ School of Electrical, Computer and Energy Engineering, Arizona State University, Tempe, AZ 85287, USA \\ wilbur.shi@asu.edu.
}

\begin{abstract}
In this paper, we consider the problem of finding a Nash equilibrium in a multi-player game over generally connected networks. This model differs from a conventional setting in that players have partial information on the actions of their opponents and the communication graph is not necessarily the same as the players' cost dependency graph. We develop a relatively fast algorithm within the framework of inexact-ADMM, based on local information exchange between the players. We prove its convergence to Nash equilibrium for fixed step-sizes and analyze its convergence rate. Numerical simulations illustrate its benefits when compared to a consensus-based gradient type algorithm with diminishing step-sizes.
\end{abstract}

Key words: Game theory over networks, distributed algorithms, noncooperative games, communication graph.

\section{Introduction}

We consider distributed Nash equilibrium (NE) seeking in a setting where players have limited local information, over a communication network. In contrast to the classical setting where all players have access to all their opponents' decisions Scutari et al. (2014), Frihauf et al. (2012), Kannan and Shanbhag (2012), our interest lies in networked regimes where agents may only access or observe the decisions of their neighbours, and there is no central node that has bidirectional communication with all players. This is a topic of increasing recent interest given the proliferation of engineering networked applications requiring distributed protocols that operate under partial, local information ( $\mathrm{V} 2 \mathrm{~V}, \mathrm{P} 2 \mathrm{P}$, ad-hoc, smartgrid networks), and the deregulation of global markets. Applications that motivate us to consider NE seeking in such a setting range from spectrum access in cognitive radio networks, Wang et al. (2010), Cheng et al. (2014), Swenson et al. (2015), rate control and congestion games in wireless, vehicular or ad-hoc networks, Alpcan and Başar (2005), Yin et al. (2011), Tekin et al. (2012), to networked Nash-Cournot competition, Bimpikis et al. (2014), Koshal et al. (2016), and opinion dynamics in social networks, Ghaderi and Srikant (2014), Bimpikis et al. (2016). For example, in cognitive radio networks users adaptively adjust their operating parameters based on interactions with the environment and other users in the network, Wang et al. (2010). In a vehicular adhoc network setting, vehicles on the road often maintain a relatively stable topology and form clusters wherein vehicles can communicate one with another. A bestresponse algorithm for channel selection is proposed in Cheng et al. (2014), based on vehicles within a cluster communicating with each other, while selfishly trying to maximize their own utilities. A similar information exchange setting is considered in Swenson et al. (2015), where because players are unable to directly observe the actions of all others, they engage in local, non-strategic information exchange to eventually agree on a common Nash equilibrium. In an economic setting, Bramoullé et al. (2014) draws attention to the problem of "who interacts with whom" in a network and to the importance of communication with neighbouring players. An example is Nash-Cournot competition played over a network, between a set of firms that compete over a set of locations, Bimpikis et al. (2014). A communication network is formed between the firms, and prescribes how they communicate locally their production decision over those locations, Koshal et al. (2016). All these examples are non-cooperative in the way decisions are made (each agent is self-interested in minimizing only its own cost), but collaborative in information sharing (agents have the incentive to exchange information with their neighbours in order to mitigate the lack of global information on 
others' decisions). Unlike the classical setting, where all others' decisions are known and information exchange is not incentive compatible, in a partial-decision information setting (not usually considered in classical game theory), information exchange is motivated because of the limited data each player has. Such an information exchange setting has started to be considered very recently, Swenson et al. (2015), Koshal et al. (2016), Salehisadaghiani and Pavel (2016), but convergence is guaranteed only for diminishing step-sizes. Motivated by the above, in this paper we develop a distributed NE seeking algorithm that operates under limited (partial) decisioninformation, and is guaranteed to converge to NE with constant step-sizes. Each player updates his decision and his estimate simultaneously in a single step, based on local information exchange with his neighbours.

We use an ADMM approach to develop the algorithm. Originally developed in 1970s, the Alternating Direction Method of Multipliers (ADMM) method has become widely used in distributed optimization problems (DOP) after its re-introduction in Boyd et al. (2011), Wei and Ozdaglar (2012), Wei and Ozdaglar (2013), Shi et al. (2014), Chang et al. (2015), Hong et al. (2016). In a DOP, $N$ agents cooperatively minimize a global objective, $f(y):=\sum_{i=1}^{N} f_{i}(y)$, over $y \in \Omega$, by communicating over a connected graph. The problem is reformulated by introducing a separate decision variable $x_{i}$ for each agent $i$ and imposing the equality constraint $x_{i}=x_{j}$ for all agents connected by an edge (neighbours), leading to

$$
\begin{cases}\underset{x}{\operatorname{minimize}} & f(x):=\sum_{i=1}^{N} f_{i}\left(x_{i}\right) \\ \text { subject to } & A x=0, \quad x_{i} \in \Omega \quad \forall i=1, \ldots, N\end{cases}
$$

where $x=\left[x_{i}\right]_{i=1}^{N}, A$ is the edge-node incidence matrix. ADMM for DOP relies on the additive structure and separability of the objective function (1) and on the linearity of the equality constraints. Each agent $i$ will minimize its cost $f_{i}\left(x_{i}\right)$ over $x_{i}$ and this $f_{i}$ is independent (decoupled) of the other agents' $x_{j}, j \neq i$. Dual decomposition leads to $N$ parallel decoupled dual ascent problems.

In this work, we exploit the benefits of ADMM in the context of finding a NE of a game, where each player (agent) $i$ aims to minimize its own cost function $J_{i}$ with respect to (w.r.t) its action $x_{i}$, given a profile of other players' actions, except himself, $x_{-i}$,

$$
\left\{\begin{array}{ll}
\underset{x_{i}}{\operatorname{minimize}} & J_{i}\left(x_{i}, x_{-i}\right) \\
\text { subject to } & x_{i} \in \Omega_{i}
\end{array} \quad \forall i=1, \ldots, N .\right.
$$

We consider a networked information exchange setting as in the motivating examples, due to players' limited (partial) information on the others' decisions. There are several challenges when comparing a game to a DOP:

- A Nash game can be seen as a set of parallel coupled optimization problems, (2). Each player's cost is dependent on the other players' decisions, hence its de- cision is directly affected by these.

- Each player $i$ updates only its own decision $x_{i}$, but he also requires an estimate of all others $\left[x_{j}\right]_{j=1, \ldots, N, j \neq i}$ or $x_{-i}$, in order to solve its optimization problem.

We introduce local estimates of players' actions and (virtual) constraints for their consensus. We reformulate the problem and relate it to a modified game (a set of optimization problems) with consensus constraints. As in classical ADMM we reduce the computational costs by using a linear approximation in players' action update rule (inexact-ADMM). However, direct application of ADMM is not possible because each minimization is not over the whole vector (estimate), but rather over part of it (action). Every player updates its decision/action as well as its estimates of the other players' actions by this synchronous, inexact ADMM-type algorithm, using its own estimates and those of its neighbours. We show that the algorithm converges to NE under fixed step-sizes, the first such algorithm to the best of our knowledge. A short version appears in Salehisadaghiani and Pavel (2017), Shi and Pavel (2017) under stronger assumptions.

Related Works. Distributed NE computation is relevant to many applications, such as sensor network coordination Stanković et al. (2012), flow control Alpcan and Başar (2005), optical networks, Pan and Pavel (2009). In the classical setting of distributed NE computation using best-response or gradient-based schemes, competitors' decisions are assumed to be observable by all players, Facchinei and Pang (2007), Frihauf et al. (2012). Recent work in the classical setting considered games with monotone pseudo-gradient Scutari et al. (2014); Kannan and Shanbhag (2012); Yousefian et al. (2016), Zhu and Frazzoli (2016), networked aggregative games with quadratic cost functions, Parise et al. (2015), or games with affine constraints, Yi and Pavel (2017, 2018). Players are assumed to have access to the others' decisions, so the issue of partial information on the opponents' decisions is not considered in the above works.

However, having access to all others' decisions can be impractical in distributed networks, Marden (2007), Li and Marden (2013). In recent years, there has been an increasing interest to consider this issue and how to deal with it via networked (partial)-information exchange. Our work is related to this literature. Consensus-based approaches have been proposed, by using fictitious play in congestion games, Swenson et al. (2015), or projectedgradient algorithms, either for the special class of $a g$ gregative games, Koshal et al. (2016), or for games where players' cost functions depend on others' actions in a general manner, in Salehisadaghiani and Pavel (2016), or are partially coupled, Salehisadaghiani and Pavel (2018). Convergence to the NE was shown only for diminishing step-sizes, under strict monotonicity of the pseudogradient. In contrast, our ADMM algorithm converges with fixed step-sizes and has faster convergence. We note that very recently continuous-time NE seeking dynamics are proposed based on consensus and gradient-type dynamics, for games with unconstrained action sets, Ye 
and $\mathrm{Hu}$ (2017), or based on passivity, Gadjov and Pavel (2018). Differently, we develop a discrete-time NE seeking algorithm for games with compact action sets, based on an ADMM approach.

Our work is related to the large literature on ADMM for DOP but there are several differences. In contrast to typical ADMM algorithms developed for DOP (1) which is separable, e.g. Wei and Ozdaglar (2012), Wei and Ozdaglar (2013), Shi et al. (2014), Chang et al. (2015), Hong et al. (2016), our ADMM algorithm for NE seeking of (2) has an extra step for estimate update, besides the update of each player's decision variable. This is because of the intrinsic coupling in $J_{i}(2)$; the update of each player's action is coupled nonlinearly to this estimate and to the others' decisions. This is unlike ADMM for DOP where, due to decoupled costs, the update of each agent's decision is only linearly coupled to the others' decisions (via the constraints). This also leads to a technical difference on the convexity assumptions under which convergence is shown. Typical assumptions in DOP are individual (strict/strong) joint convexity of each agent's decoupled cost function $f_{i}$ (1), Wei and Ozdaglar (2012), Wei and Ozdaglar (2013), Shi et al. (2014), Chang et al. (2015). In the augmented space, due to separability of DOP (1), monotonicity of the full gradient is automatically maintained. Different from that, in a game setup, individual joint convexity is too restrictive, unless the game is separable to start with, which is a trivial case. Rather, a typical assumption is individual partial convexity of each cost, and monotonicity (strict, strong) of the pseudo-gradient. Furthermore, in the augmented space of actions and estimates, due to the inherent coupling in (2), monotonicity is not necessarily maintained. We note that in ADMM for DOP (1), relaxation from strongly convex to merely convex $f_{i}$ involves either an exact augmented minimization at each step, (hence $f_{i}$ needs to have inexpensive proximal-operator), Wei and Ozdaglar (2013), or an extra quadratic augmentation, for a sufficiently large step-size, Hong et al. (2016). In ADMM for game (2), we use a similar idea as Hong et al. (2016) for the extended pseudo-gradient (which may be non-monotone in the augmented space). However, unlike Hong et al. (2016), since cost functions $J_{i}$ are not separable, our analysis is novel, based on the decomposition of the augmented space into the consensus subspace and its orthogonal complement. Moreover, our proof techniques rely on the pseudo-gradient, since we cannot employ a common Lagrangian as in DOP. Another consequence is that, unlike Wei and Ozdaglar (2013), we are able to characterize the rate of convergence only in terms of the residuals relative to NE optimality.

The paper is organized as follows. The problem statement and assumptions are provided in Section 2. In Section 3, an inexact ADMM-type algorithm is developed. Its convergence to Nash equilibrium is analyzed in Section 4. Simulation results are given in Section 5 and conclusions in Section 6.

\subsection{Notations and Background}

A vector $x \in \mathbb{R}^{n}$ may be represented in equivalent ways as $x=\left[x_{1}, \ldots, x_{n}\right]^{T}=\left[x_{1} ; \ldots ; x_{n}\right]$, or $x=\left[x_{i}\right]_{i=1, \ldots, n}$, or $x=\left(x_{i}, x_{-i}\right)$. All vectors are assumed to be column vectors. Given a vector $x \in \mathbb{R}^{n}, x^{T}$ denotes its transpose and $\|x\|=\sqrt{x^{T} x}$ denotes its Euclidean norm. Given a symmetric $n \times n$ matrix $A,\|x\|_{A}$ denotes the weighted norm $\|x\|_{A}:=\sqrt{x^{T} A x}$. Denote $\mathbf{1}_{n}=[1,1, \ldots, 1]^{T} \in \mathbb{R}^{n}$ and $\mathbf{0}_{n}=[0,0, \ldots, 0]^{T} \in \mathbb{R}^{n}$. Let $e_{i}$ denote the $N \times 1, i$ th unit vector in $\mathbb{R}^{n}$, whose $i$-th element is 1 and the rest are 0 . Given a vector $x \in \mathbb{R}^{n}, \operatorname{diag}\left(\left[x_{i}\right]_{i=1, \ldots, n}\right)$ denotes the $n \times n$ diagonal matrix with $x_{1}, \ldots, x_{n}$ on the diagonal. Similarly, given $A_{i}, i=1, \ldots, n$ as $(p \times q)$ matrices, $\operatorname{diag}\left(\left[A_{i}\right]_{i=1, \ldots, n}\right)$ denotes the $(n p \times n q)$ block-diagonal matrix with $A_{i}$ on the diagonal. $I_{n}$ denotes the identity matrix in $\mathbb{R}^{n \times n}$. The Kronecker product of matrices $\mathrm{A}$ and $\mathrm{B}$ is denoted by $A \otimes B$. Given matrices $A, B \in \mathbb{R}^{n \times n}$, $A \succ 0(A \succeq 0)$ denotes that $A$ is positive (semi-)definite. $\lambda_{\min }(A)$ and $\lambda_{\max }(A)$ represent the minimum and the maximum eigenvalue of matrix $A$, respectively. Given a $m \times n$ matrix $A,\|A\|_{2}$ denotes its induced 2-norm, $\|A\|_{2}=\sigma_{\max }(A), \sigma_{\max }(A)=\sqrt{\lambda_{\max }\left(A^{T} A\right)}$. For any $a, b \in \mathbb{R}^{n}$ and $\rho>0$,

$$
-\frac{1}{2 \rho}\|a\|^{2}-\frac{\rho}{2}\|b\|^{2} \leq a^{T} b \leq \frac{1}{2 \rho}\|a\|^{2}+\frac{\rho}{2}\|b\|^{2} .
$$

For every $a, b, c \in \mathbb{R}^{n}$ and $n \times n$ matrix $A \succeq 0$,

$(a-b)^{T} A(a-c)=\frac{1}{2}\|a-c\|_{A}^{2}+\frac{1}{2}\|a-b\|_{A}^{2}-\frac{1}{2}\|b-c\|_{A}^{2}$.

Given a set $\Omega \in \mathbb{R}^{n},|\Omega|$ denotes the cardinality of $\Omega$. The Euclidean projection of $x \in \mathbb{R}^{n}$ onto $\Omega \subset \mathbb{R}^{n}$ is denoted by $T_{\Omega}\{x\}$. Denote by $\operatorname{prox}_{g}^{a}$ the proximal operator for function $g$ with a constant $a$, defined as follows:

$$
\operatorname{prox}_{g}^{a}\{s\}:=\arg \min _{x}\left\{g(x)+\frac{a}{2}\|x-s\|^{2}\right\} .
$$

Let $\mathcal{I}_{\Omega}(x):=\left\{\begin{array}{ll}0 & \text { if } x \in \Omega \\ \infty & \text { otherwise }\end{array}\right.$, be the indicator function of a closed convex set $\Omega \in \mathbb{R}^{n}$. Then, $\operatorname{prox}_{\mathcal{I}_{\Omega}}^{a}\{\cdot\}=T_{\Omega}\{\cdot\}$. Let $\partial \mathcal{I}_{\Omega}(x)$ denote the subdifferential of $\mathcal{I}_{\Omega}$ at $x$, i.e., the set of all subgradients of $\mathcal{I}_{\Omega}$ at $x$. Then $\partial \mathcal{I}_{\Omega}(x)$ is a convex set and $\partial \mathcal{I}_{\Omega}(x)=N_{\Omega}(x)$, where $N_{\Omega}(x)=\{y \in$ $\left.\mathbb{R}^{n} \mid y^{T}\left(x^{\prime}-x\right) \leq 0, \forall x^{\prime} \in \Omega\right\}$ is the normal cone to $\Omega$ at $x$. Moreover, $\left(y_{1}-y_{2}\right)^{T}\left(x_{1}-x_{2}\right) \geq 0, \forall x_{1}, x_{2} \in \Omega$, $\forall y_{1} \in \partial \mathcal{I}_{\Omega}\left(x_{1}\right), \forall y_{2} \in \partial \mathcal{I}_{\Omega}\left(x_{2}\right)$, Rockafellar (1970).

For an undirected graph $G(V, E)$, we denote by:

- $V$ : Set of vertices in $G$,

- $E \subseteq V \times V$ : Set of all edges in $G$. $(i, j) \in E$ if and only if $i$ and $j$ are connected by an edge,

- $N_{i}:=\{j \in V \mid(i, j) \in E\}$ : Set of neighbours of $i$ in $G$,

- $A:=\left[a_{i j}\right]_{i, j \in V}:$ Adjacency matrix of $G$ where $a_{i j}=1$ if $(i, j) \in E$ and $a_{i j}=0$ otherwise,

- $\mathbb{D}:=\operatorname{diag}\left(\left|N_{1}\right|, \ldots,\left|N_{N}\right|\right)$ : Degree matrix of $G, d=$ $\operatorname{trace}(\mathbb{D})=\sum_{i=1}^{N}\left|N_{i}\right|, d^{*}=\max _{i}\left\{\left|N_{i}\right|\right\}$,

- $L: \mathbb{D}-A$ : Laplacian matrix of $G$, 
- $L_{N}:=\mathbb{D}^{-\frac{1}{2}} L \mathbb{D}^{-\frac{1}{2}}:$ Normalized Laplacian of $G$ if $G$ has no isolated vertex.

The following hold for a graph $G$ with no isolated vertex: $\mathbb{D} \succ 0, \lambda_{\max }\left(L_{N}\right) \leq 2$ and $2 \mathbb{D}-L=\mathbb{D}^{\frac{1}{2}}\left(2 I-L_{N}\right) \mathbb{D}^{\frac{1}{2}} \succeq 0$, Chung (1997). For a connected, undirected graph $G$ with $n$ vertices, $L \succeq 0$, with 0 a simple eigenvalue and $L \mathbf{1}_{n}=$ $\mathbf{0}_{n}, \mathbf{1}_{n}^{T} L=\mathbf{0}_{n}^{\bar{T}}$. The other eigenvalues of $L$ are positive, with minimum one $\lambda_{2}(L)$ and maximum $\lambda_{\max }(L) \leq 2 d^{*}$. Also, $\operatorname{Ker}(L)=\operatorname{span}\left\{\mathbf{1}_{n}\right\}, \operatorname{Ker}(L)^{\perp}=\left\{x \mid \mathbf{1}_{n}^{T} x=0\right\}$, $x^{T} L x \geq \lambda_{2}(L)\|x\|^{2}>0, \forall x \neq 0, x \in \operatorname{Ker}(L)^{\perp}$.

\section{Problem Statement}

Consider a networked game with $N$ players, defined with the following parameters and denoted by $\mathcal{G}\left(V, \Omega_{i}, J_{i}\right)$ :

- $V=\{1, \ldots, N\}$ : Set of all players,

- $\Omega_{i} \subset \mathbb{R}$ : Action set of player $i, \forall i \in V$,

- $\Omega=\prod_{i \in V} \Omega_{i} \subset \mathbb{R}^{N}$ : Action set of all players, where $\prod$ denotes the Cartesian product,

- $J_{i}: \mathbb{R}^{N} \rightarrow \mathbb{R}$ : Cost function of player $i, \forall i \in V$.

Players' actions are denoted as follows:

- $x_{i} \in \Omega_{i}$ : Player $i$ 's action, $\forall i \in V$,

- $x_{-i} \in \Omega_{-i}:=\prod_{j \in V \backslash\{i\}} \Omega_{j}$ : All players' actions except player $i$ 's,

- $x=\left(x_{i}, x_{-i}\right) \in \Omega$ : All players actions.

The game is played such that for a given $x_{-i} \in \Omega_{-i}$, every player $i \in V$ aims to minimize its own cost function (2) with respect to (w.r.t.) $x_{i}$. Note that each player's optimal action is dependent on the other players' decisions. A Nash equilibrium (NE) lies at the intersection of solutions to the set of problems (2) (fixed-point of bestresponse map), such that no player can reduce its cost by unilaterally deviating from its action.

Definition 1 Consider an $N$-player game $\mathcal{G}\left(V, \Omega_{i}, J_{i}\right)$. An action profile (vector) $x^{*}=\left(x_{i}^{*}, x_{-i}^{*}\right) \in \Omega$ is called a Nash equilibrium (NE) of this game if

$$
J_{i}\left(x_{i}^{*}, x_{-i}^{*}\right) \leq J_{i}\left(x_{i}, x_{-i}^{*}\right) \quad \forall x_{i} \in \Omega_{i}, \forall i \in V .
$$

We state a few assumptions for the existence of a NE, Nash (1950); Debreu (1952); Glicksberg (1952).

Assumption 1 For every $i \in V$,

- $\Omega_{i} \subset \mathbb{R}$ is non-empty, compact and convex,

- $J_{i}\left(x_{i}, x_{-i}\right)$ is $C^{1}$ and convex in $x_{i}$, for every $x_{-i}$, and jointly continuous in $x$.

Let $\nabla_{i} J_{i}(x)=\frac{\partial J_{i}}{\partial x_{i}}\left(x_{i}, x_{-i}\right)$ be the partial gradient of $J_{i}$ w.r.t. $x_{i}$ and $F: \mathbb{R}^{N} \rightarrow \mathbb{R}^{N}$ be the pseudo-gradient of game (2) defined by

$$
F(x):=\left[\nabla_{i} J_{i}(x)\right]_{i \in V}
$$

Let $\partial \mathcal{I}_{\Omega_{i}}\left(x_{i}\right)$ be the subdifferential of $\mathcal{I}_{\Omega_{i}}$ at $x_{i}$ and $G(x):=\left[\partial \mathcal{I}_{\Omega_{i}}\left(x_{i}\right)\right]_{i \in V}$, where $\partial \mathcal{I}_{\Omega_{i}}\left(x_{i}\right)=N_{\Omega_{i}}\left(x_{i}\right)$. Let $N_{\Omega}(x)=\prod_{i \in V} N_{\Omega_{i}}\left(x_{i}\right)$ be the normal cone to $\Omega$ at $x$.

A Nash equilibrium (NE) $x^{*}=\left(x_{i}^{*}, x_{-i}^{*}\right) \in \Omega$ satisfies the variational inequality, (cf. Proposition 1.4.2, Facchinei and Pang (2007))

$$
\begin{aligned}
& \left(x-x^{*}\right)^{T} F\left(x^{*}\right) \geq 0, \forall x \in \Omega, \\
\text { i.e., }-F\left(x^{*}\right) & \in N_{\Omega}\left(x^{*}\right) \text {, or } \\
- & -\nabla_{i} J_{i}\left(x^{*}\right) \in N_{\Omega_{i}}\left(x_{i}^{*}\right), \forall i \in V,
\end{aligned}
$$

Thus, with $\partial \mathcal{I}_{\Omega_{i}}\left(x_{i}\right)=N_{\Omega_{i}}\left(x_{i}\right)$, a NE $x^{*}=\left(x_{i}^{*}, x_{-i}^{*}\right)$ of (2) can be characterized by

$$
0 \in \nabla_{i} J_{i}\left(x^{*}\right)+\partial \mathcal{I}_{\Omega_{i}}\left(x_{i}^{*}\right), \forall i \in V,
$$

Then, with (6), (7) can be written in compact form as,

$$
\mathbf{0}_{N} \in F\left(x^{*}\right)+G\left(x^{*}\right) \text {. }
$$

Typically another assumption such as monotonicity (strict, strong) of the pseudo-gradient vector $F,(6)$, is used to show that projected-gradient type algorithms converge to $x^{*}$, Facchinei and Pang (2007).

Assumption 2 The pseudo-gradient $F$ is strongly monotone and Lipschitz continuous: there exists $\mu>0$, $\theta_{0}>0$ such that for any $x$ and $y,\langle x-y, F(x)-F(y)\rangle \geq$ $\mu\|x-y\|^{2}$ and $\|F(x)-F(y)\| \leq \theta_{0}\|x-y\|$.

Under Assumptions 1 and 2, the game has a unique NE $x^{*}$ (Theorem 2.3.3 in Facchinei and Pang (2007)). Strong monotonicity of $F$ is a standard assumption under which convergence of projected-gradient type algorithms is guaranteed with fixed step-sizes, (Theorem 12.1.2 in Facchinei and Pang (2007)).

We assume that the cost function $J_{i}$ and the action set $\Omega_{i}$ information are available to player $i$ only, hence an incomplete-information game, Li and Başar (1987). The challenge is that each optimization problem in (2) is dependent on the solution of the other simultaneous problems. In the classical setting of a game with incomplete information but perfect monitoring, each agent can observe the actions of all other players, $x_{-i}$, Facchinei and Pang (2007), Scutari et al. (2014), Frihauf et al. (2012), Kannan and Shanbhag (2012). In this paper, we consider a game with incomplete information and imperfect monitoring, where no player is able to observe the actions of all others. We refer to this as a game with partial-decision information. To compensate for the lack of global decision information, players exchange some information in order to update their actions. We assume players can communicate only locally, with their neighbours. An undirected communication graph $G_{c}(V, E)$ is then defined with no isolated vertex. Let denote the set of neighbours of player $i$ in $G_{c}$ by $N_{i}$. Let also denote $\mathbb{D}$ and $L$ be the degree and Laplacian matrices associated to $G_{c}$, respectively. The following assumption is used.

Assumption $3 G_{c}$ is undirected and connected.

We assume that players maintain estimates of the other players' actions and share them with their neighbours in order to update their estimates. In an engineering application, this step could be prescribed, e.g. as a network protocol in peer-to-peer networks or ad-hoc networks. Our goal is to develop a distributed algorithm for computing the NE of $\mathcal{G}\left(V, \Omega_{i}, J_{i}\right)$ with fixed step-sizes, while 


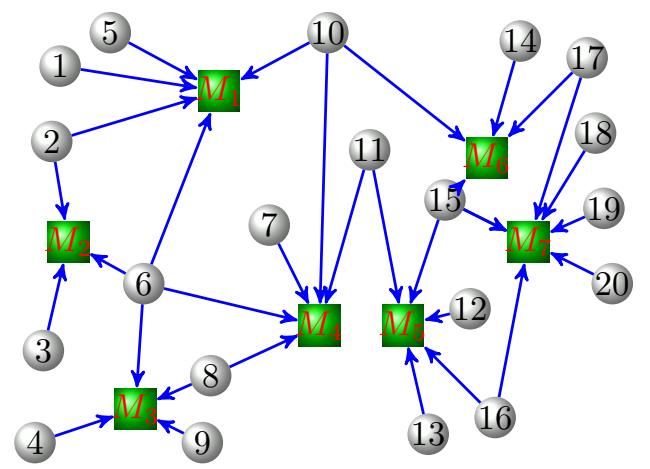

Figure 1. Network Cournot game: An edge from $i$ to $M_{k}$ on this graph implies that agent/firm $i$ participates in Market $M_{k}$.

using only partial-decision information over the communication graph $G_{c}(V, E)$.

This is a topic of recent interest given the the proliferation of engineering networked applications requiring distributed protocols that operate under partial, local information (e.g. ad-hoc networks or smart-grid networks), and the deregulation of global markets. We provide next a motivating example, inspired by the one in Koshal et al. (2016), Kannan and Shanbhag (2012).

Example 1 (Nash-Cournot Game Over a Network). Consider a networked Nash-Cournot game, as in Koshal et al. (2016), Bimpikis et al. (2014) between a set of $N$ firms (players/agents) involved in the production of a homogeneous commodity that compete over $m$ markets, $M_{1}, \cdots, M_{m}$ (Figure 1). Firm $i, i \in V$ participates in $n_{i} \leq m$ markets with $x_{i}$ commodity amount that it supplies to each of its markets. Hence player $i$ 's action (strategy) is its commodity amount per market $x_{i} \in \mathbb{R}$. Its total amount it produces is $x_{i, T}=n_{i} x_{i}$, assumed to be limited to $\Omega_{T}$, so that $x_{i} \in \Omega_{i} \subset \mathbb{R}, \Omega_{i}=\Omega_{T} / n_{i}$. Firm $i$ has a local vector $A_{i} \in \mathbb{R}^{m}$ (with elements 1 or 0 ) that specifies which markets it participates in. The $k$-th element of $A_{i}$ is 1 if and only if player $i$ delivers $x_{i}$ amount to market $M_{k}$. Therefore, $A_{1}, \cdots, A_{N}$ can be used to specify a bipartite graph that represents the connections between firms and markets (see Figure 1). Denote $x=\left[x_{i}\right]_{i \in V} \in \mathbb{R}^{N}$, and $A=\left[A_{1}, \cdots, A_{N}\right] \in \mathbb{R}^{m \times N}$. Then $A x \in \mathbb{R}^{m}=\sum_{i=1}^{N} A_{i} x_{i}$ is the total product supply to all markets, given the action profile $x$ of all firms. Suppose that $P: \mathbb{R}^{m} \rightarrow \mathbb{R}^{m}$ is a price vector function that maps the total supply of each market to the corresponding market's price. Each firm $i$ has a production cost $c_{i}\left(x_{i, T}\right): \Omega_{T} \rightarrow \mathbb{R}$, function of its total production amount. Then the local objective function of firm (player) $i$ is $J_{i}\left(x_{i}, x_{-i}\right)=c_{i}\left(x_{i, T}\right)-P^{T}(A x) A_{i} x_{i}$. Overall, given the other firms' profile $x_{-i}$, each firm needs to solve the following optimization problem,

$$
\underset{x_{i} \in \Omega_{i}}{\operatorname{minimize}} \quad c_{i}\left(x_{i, T}\right)-P^{T}(A x) A_{i} x_{i}
$$

In the classical, centralized-information setting each firm is assumed to have instantaneous access to the others'

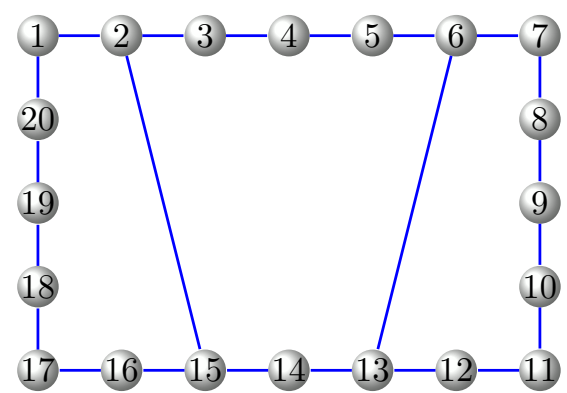

Figure 2. Communication graph $G_{c}$ : Firms $i$ and $j$ are able to exchange their local $x^{i}$ and $x^{j}$ if there exists an edge between them on this graph.

actions $x_{-i}$. This may be impractical in a large network of geographically distributed firms, Bimpikis et al. (2014). For example, consider that Figure 1 depicts a group of $N=20$ firms located on different continents that participate in $m=7$ markets, with no centralized communication system between them. Since players are unable to directly observe the actions of all others, they engage in local, non-strategic information exchange, to mitigate their lack of global, centralized information. Firms/players may communicate with a local subset of neighbouring firms via some underlying communication infrastructure, hence a distributed partial-information setting. A communication network formed $G_{c}$ between the firms prescribes how they communicate locally their production decision, Koshal et al. (2016). In this situation, the communication network is formed by the players who are viewed as the nodes in the network. In this example, we consider that most of the communication is between firms on the same continent, with one or at most two firms in each continent having a direct connection to another firm on another continent. One such instance of the communication network $G_{c}$ is shown in Figure 2. Firms $i$ and $j$ are able to exchange their local $x^{i}$ and $x^{j}$ if there exists an edge between them on this graph. Various other topologies can be considered, with different connectivity.

A Nash-Cournot game (9) can describe other practical decision problems in engineering networks, for example rate control games in communication networks (Yin et al. (2011)) and demand-response games in smart-grid networks (Ye and Hu (2017), Yi and Pavel (2018)). Another example, of a rate control game over a wireless ad-hoc network, is presented in Section 5 .

\section{Distributed Inexact-ADMM Algorithm}

In order to find the NE of $\mathcal{G}\left(V, \Omega_{i}, J_{i}\right)(2)$ over the communication graph $G_{c}(V, E)$, we propose a distributed ADMM-type algorithm with fixed step-sizes (Bertsekas and Tsitsiklis (1997), p. 255), based on introducing local estimates of players' actions and using (virtual) constraints for estimate consensus.

We define a few notations for players' estimates. 
- $x_{-i}^{i} \in \mathbb{R}^{N-1}$ : Player $i$ 's estimate of all other players' actions,

- $x_{i}^{i} \in \Omega_{i} \subset \mathbb{R}$ : Player $i$ 's estimate of its action which is indeed its own action, i.e., $x_{i}^{i}=x_{i}$ for $i \in V$,

- $x^{i}=\left(x_{i}^{i}, x_{-i}^{i}\right) \in \mathbb{R}^{N}$ : Player $i$ 's estimate of all players' actions (state variable),

- $\mathbf{x}=\left[x^{i}\right]_{i \in V}$ or $\mathbf{x}=\left[x^{1} ; \ldots ; x^{N}\right] \in \mathbb{R}^{N^{2}}:$ Augmented (stacked) vector of all estimates.

Note that all players' actions $x$ can be represented as $x=\left[x_{i}^{i}\right]_{i \in V}$. In steady-state all local copies $x^{i}$ should be equal. By employing the actions' estimates $x^{i}, \forall i \in V$, (local copies of $x$ ), we can reformulate game (2) as:

$$
\begin{cases}\underset{x_{i}^{i} \in \Omega_{i}}{\operatorname{minimize}} & J_{i}\left(x_{i}^{i}, x_{-i}^{i}\right), \quad \forall i \in V \\ \text { subject to } & x^{i}=x^{j}, \forall j \text { s.t. }(i, j),(j, i) \in E\end{cases}
$$

where $E$ is the set of edges of $G_{c}$. For each agent $i$ we consider the constraint that the estimate (local copy) $x^{i}$ is equal to $x^{j}$ of its neighbours for all cases where $x^{i}$ is involved, i.e., player $i$ is either a source or a destination, as specified via ordered pairs $(i, j) \in E$ and $(j, i) \in E$. Under Assumption 3 this ensures that $x^{1}=x^{2} \ldots=$ $x^{N}$, hence (10) recovers (2). The following distributed ADMM-type algorithm is proposed.

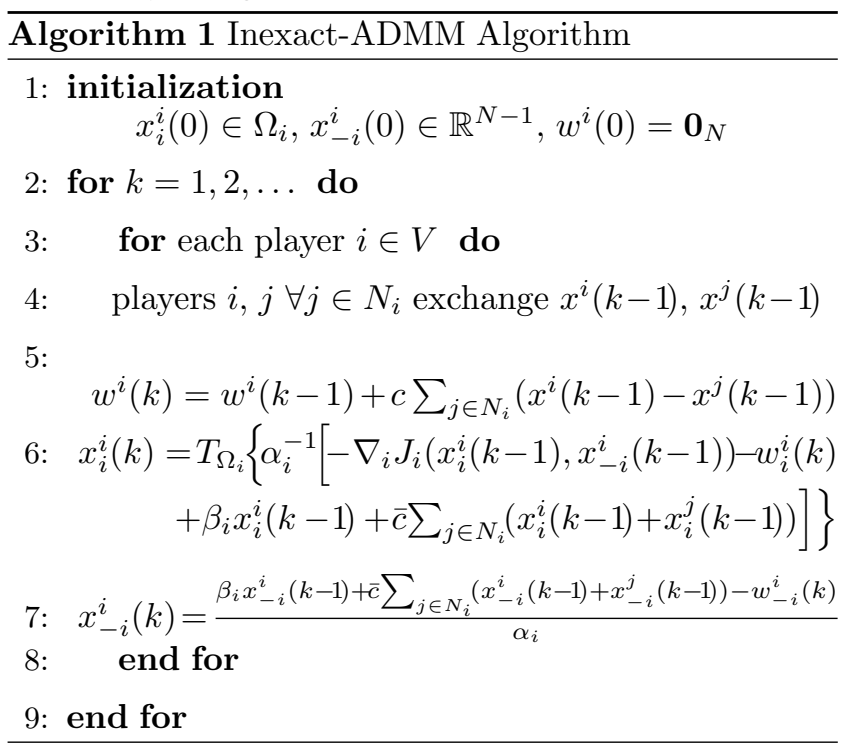

Here $\alpha_{i}=\beta_{i}+2 \bar{c}\left|N_{i}\right|, \quad \bar{c}=c+c_{0}$, where $c>0, c_{0}>0$, $\beta_{i}>0$ are penalty parameters for the augmented Lagrangian and proximal first-order approximation. Besides its estimates, each player $i$ needs to maintain only its dual variables $w^{i}$, and needs only the information on its $\Omega_{i}$ set. The derivation of Algorithm 1 following an ADMM approach adapted to a game setup is provided in Appendix A.

Algorithm 1 has a gradient-play structure for a game with estimate consensus constraints: Step 6 uses a projected-gradient (due to $J_{i}$ ) of an augmented Lagrangian with quadratic-penalty and proximal linearization (inexact ADMM) (see (A.10) in Appendix A). So in this sense, in the context of individual rationality, it can be interpreted as a better-response (as opposed to best-response) strategy, but in the modified game, due to partial-information setting. Step 5 updates the dual variables $w^{i}$ via a dual ascent. Compared to a gossipbased algorithm, Koshal et al. (2016), Salehisadaghiani and Pavel (2016), the additional ADMM-induced modifications are instrumental to prove convergence under fixed step-sizes.

Remark 1 We compare Algorithm 1 to typical ADMM DOP algorithms. Unlike Wei and Ozdaglar (2012), Wei and Ozdaglar (2013), where a minimization subproblem is solved exactly at each iteration, Algorithm 1 uses a gradient update based on inexact (proximal) approximation as in Hong et al. (2016). In contrast to either of these algorithms, the update of each player decision $x_{i}^{i}$ (Step 6) is indirectly coupled nonlinearly to the previous decisions of other players, via its estimate $x_{-i}^{i}$, due to the intrinsic coupling in $J_{i}$. This is unlike ADMM for DOP where the update of each agent's decision variable $x_{i}$ is only linearly coupled to the others agents' $x_{j}$. Moreover, in Algorithm 1 each player has an extra step for updating its estimate $x_{-i}^{i}$ (Step 7), in addition to its decision variable $x_{i}^{i}$. Because of this coupling the convergence proof (based on the pseudo-gradient) is more involved.

Next we analyze the fixed points of Algorithm 1.

Lemma 1 Consider that Assumptions 1, 3 hold. Let $\overline{\mathbf{x}}:=\left[\bar{x}^{i}\right]_{i \in V}, \overline{\mathbf{w}}:=\left[\bar{w}^{i}\right]_{i \in V}$ be any fixed points of Algorithm 1. Then, $\overline{\mathbf{x}}=\mathbf{1}_{N} \otimes \bar{x}$, where $\bar{x}=x^{*}$ is NE of game $(2)$, and $\left(\mathbf{1}_{N}^{T} \otimes I_{N}\right) \overline{\mathbf{w}}=\mathbf{0}_{N}$.

Proof. From Step 5 of Algorithm 1, for all $i \in V$,

$$
\bar{w}^{i}=\bar{w}^{i}+c \sum_{j \in N_{i}}\left(\bar{x}^{i}-\bar{x}^{j}\right) \Rightarrow\left(L \otimes I_{N}\right) \overline{\mathbf{x}}=\mathbf{0}_{N^{2}}
$$

and by Assumption 3, this yields $\overline{\mathbf{x}}=\mathbf{1}_{N} \otimes \bar{x}$, for some $\bar{x}=\left[\bar{x}_{i}\right]_{i \in V} \in \mathbb{R}^{N}$, hence

$$
\bar{x}^{1}=\ldots=\bar{x}^{N}=\bar{x},
$$

Using (11) in Step 6 of Algorithm 1 yields, $\left(\beta_{i}+\right.$ $\left.2 \bar{c}\left|N_{i}\right|\right) \bar{x}_{i}-\alpha_{i} \bar{x}_{i} \in \nabla_{i} J_{i}(\bar{x})+\partial \mathcal{I}_{\Omega_{i}}\left(\bar{x}_{i}\right)+\bar{w}_{i}^{i}$. With $\alpha_{i}=\beta_{i}+2 \bar{c}\left|N_{i}\right|$ this leads to

$$
0 \in \nabla_{i} J_{i}(\bar{x})+\partial \mathcal{I}_{\Omega_{i}}\left(\bar{x}_{i}\right)+\bar{w}_{i}^{i}, \quad \forall i \in V
$$

Moreover, using (11) in Step 7 of Algorithm 1, we obtain $\alpha_{i} \bar{x}_{-i}^{i}=\alpha_{i} \bar{x}_{-i}^{i}-\bar{w}_{-i}^{i}$, hence

$$
\bar{w}_{-i}^{i}=\mathbf{0}_{N-1} \forall i \in V
$$

Combining the previous two relations into a single vector, yields

$$
\mathbf{0}_{N} \in \nabla_{i} J_{i}(\bar{x}) e_{i}+\partial \mathcal{I}_{\Omega_{i}}\left(\bar{x}_{i}\right) e_{i}+\bar{w}^{i}, \quad \forall i \in V
$$

where $e_{i}$ is the $i$-th unit vector in $\mathbb{R}^{N}$. Summing after $i \in V$, with $F(\bar{x})=\left[\nabla_{i} J_{i}(\bar{x})\right]_{i \in V}$, yields

$$
\mathbf{0}_{N} \in F(\bar{x})+G(\bar{x})+\sum_{i \in V} \bar{w}^{i} \text {. }
$$


For $w^{i}(k)$, from Step 5 of Algorithm 1, it follows that

$$
\sum_{i \in V} w^{i}(k)=\sum_{i \in V} w^{i}(k-1)+c \sum_{i \in V} \sum_{j \in N_{i}}\left(x^{i}(k-1)-x^{j}(k-1)\right)
$$

Under Assumption 3 the second term in (14) is zero, hence $\sum_{i \in V} w^{i}(k)=\sum_{i \in V} w^{i}(k-1)$ for any $k \geq 1$. With initial conditions $w^{i}(0)=\mathbf{0}_{N} \forall i \in V$ this implies that $\sum_{i \in V} w^{i}(k)=\mathbf{0}_{N} \forall k \geq 1$, hence $\sum_{i \in V} \bar{w}^{i}=\mathbf{0}_{N}$. Thus (13) reduces to $\mathbf{0}_{N} \in F(\bar{x})+G(\bar{x})$, hence by (8) $\bar{x}=x^{*}$ is $\mathrm{NE}$ and $\overline{\mathbf{x}}=\mathbf{1}_{N} \otimes x^{*}$. Moreover,

$$
\left(\mathbf{1}_{N}^{T} \otimes I_{N}\right) \overline{\mathbf{w}}=\sum_{i \in V} \bar{w}^{i}=\mathbf{0}_{N}
$$

Recall the pseudo-gradient (6), $F(x)=\left[\nabla_{i} J_{i}(x)\right]_{i \in V}$.

Definition 2 Let $\mathbf{F}: \mathbb{R}^{N^{2}} \rightarrow \mathbb{R}^{N}$, be the extension of $F$ to the augmented space, defined as

$$
\mathbf{F}(\mathbf{x}):=\left[\nabla_{i} J_{i}\left(x^{i}\right)\right]_{i \in V}, \nabla_{i} J_{i}\left(x^{i}\right)=\frac{\partial J_{i}}{\partial x_{i}^{i}}\left(x_{i}^{i}, x_{-i}^{i}\right)
$$

and called the extended pseudo-gradient. Let also $\mathbf{G}(\mathbf{x}):=\left[\partial I_{\Omega_{i}}\left(x_{i}^{i}\right)\right]_{i \in V}$, so $\mathbf{G}(\mathbf{x})=G(x)$.

Note that $\mathbf{F}$ is the pseudo-gradient evaluated at estimates instead of actual actions, and $\mathbf{F}\left(\mathbf{1}_{N} \otimes x\right)=F(x)$. We write Algorithm 1 in a compact vector form.

Proposition 1 Let $\mathbf{x}=\left[x^{i}\right]_{i \in V}, \mathbf{w}=\left[w^{i}\right]_{i \in V}$. Then the updates in Algorithm 1 have the following vector form:

$$
\begin{aligned}
\mathbf{w}(k) & =\mathbf{w}(k-1)+c \mathbf{L x}(k-1) \\
\mathbf{0}_{N^{2}} & \in \mathbf{R}(\mathbf{F}(\mathbf{x}(k-1))+\mathbf{G}(\mathbf{x}(k)))+\mathbf{w}(k) \\
& +\left((\mathbb{B}+2 \bar{c} \mathbb{D}) \otimes I_{N}\right)(\mathbf{x}(k)-\mathbf{x}(k-1))+\bar{c} \mathbf{L x}(k-1),
\end{aligned}
$$

where $\mathbf{R}=\operatorname{diag}\left(\left[e_{i}\right]_{i \in V}\right)$ is $\left(N^{2} \times N\right)$ block-diagonal matrix with unit vectors $e_{i}, \mathbf{L}=L \otimes I_{N}, \mathbf{F}, \mathbf{G}$ as in $(16), \mathbb{D}$ is the degree matrix of $G_{c}, L$ is the Laplacian matrix of $G_{c}, \mathbb{B}:=\operatorname{diag}\left(\left(\beta_{i}\right)_{i \in V}\right)$ with $\beta_{i}>0, \bar{c}=c+c_{0}, c, c_{0}>0$.

Proof. See Appendix B.

Properties of $\mathbf{F}$ and matrices $\mathbf{R}$ and $\mathbf{L}$ play a key role in the following. Matrix $\mathbf{R}^{T}$ allows action selection from the stacked $\mathbf{x}: \mathbf{R}^{T} \mathbf{x}=\left[e_{i}^{T} x^{i}\right]_{i \in V}=x$, based on $e_{i}^{T} x^{i}=$ $x_{i}^{i}=x_{i}$. Also $\left(\mathbf{1}_{N}^{T} \otimes I_{N}\right) \mathbf{R}=I_{N}, \mathbf{R}^{T} \mathbf{R}=I_{N}$. For matrix $\mathbf{L}, \operatorname{Ker}(\mathbf{L})=\operatorname{Ker}\left(L \otimes I_{N}\right)=\operatorname{span}\left\{\mathbf{1}_{N} \otimes I_{N}\right\}$ (the consensus subspace), and $\operatorname{Ker}(\mathbf{L})^{\perp}=\operatorname{span}(\mathbf{L})=\operatorname{Ker}\left\{\mathbf{1}_{N}^{T} \otimes I_{N}\right\}$.

Note that by Lemma $1, \overline{\mathbf{x}}=\mathbf{1}_{N} \otimes \bar{x} \in \operatorname{Ker}(\mathbf{L})$ and $\overline{\mathbf{w}} \in \operatorname{span}(\mathbf{L})($ cf. (15)). Also, we can write (12) as,

$$
\mathbf{0}_{N^{2}} \in\left[\nabla_{i} J_{i}(\bar{x}) e_{i}\right]_{i \in V}+\left[\partial \mathcal{I}_{\Omega_{i}}\left(\bar{x}_{i}\right) e_{i}\right]_{i \in V}+\overline{\mathbf{w}}
$$

or, with $\mathbf{F}, \mathbf{G}$ as in (16), compactly as,

$$
\mathbf{0}_{N^{2}} \in \mathbf{R}(\mathbf{F}(\overline{\mathbf{x}})+\mathbf{G}(\overline{\mathbf{x}}))+\overline{\mathbf{w}} .
$$

\section{Convergence Analysis}

In this section we show global convergence of Algorithm 1 under the following assumption.
Assumption 4 The extended pseudo-gradient $\mathbf{F}$, (16), Re Lipschitz gontinuous: there exists $\theta \overrightarrow{\mathrm{F}}$ is such that for

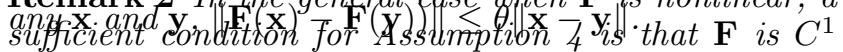
with bounded Jacobian $\|D \mathbf{F}(\mathbf{x})\|_{2}$. For quadratic games, Assumption 4 is automatically satisfied ( $\mathbf{F}$ is linear). Moreover, as shown below, $\theta=\theta_{0}$, where $\theta_{0}$ is the Lipschitz constant of $F$ (Assumption 2). Consider the Nash-Cournot game in Example 1, where firm i's production cost is a strongly convex quadratic function in its total production amount, $c_{i}\left(x_{i, T}\right)=q_{i} x_{i, T}^{2}+b_{i} x_{i, T}$, i.e., $c_{i}\left(x_{i}\right)=n_{i}^{2} q_{i} x_{i}^{2}+n_{i} b_{i} x_{i}$, where $q_{i}>0, b_{i} \in \mathbb{R}$. Consider that market $M_{k}$ 's price is a linear function of the total supplied commodity amount, $p_{k}(x)=\bar{P}_{k}-z_{k}[A x]_{k}$ (known as a linear inverse demand function) with $\bar{P}_{k}, z_{k}>0$. Denote $P=\left[p_{k}\right]_{k=1, m}: \mathbb{R}^{N} \rightarrow \mathbb{R}^{m}$, $\bar{P}=[\bar{P}]_{k=1, m} \in \mathbb{R}^{m}, Z=\operatorname{diag}\left(\left[z_{k}\right]_{k=1, m}\right) \in \mathbb{R}^{m \times m}$, so that $P=\bar{P}-Z A x$ is the vector price function, hence the objective function of player $i$ is

$$
\begin{gathered}
J_{i}\left(x_{i}, x_{-i}\right)=c_{i}\left(x_{i}\right)-(\bar{P}-Z A x)^{T} A_{i} x_{i} \text { and } \\
\nabla_{i} J_{i}(x)=\nabla c_{i}\left(x_{i}\right)+A_{i}^{T} Z A_{i} x_{i}-A_{i}^{T}(\bar{P}-Z A x),
\end{gathered}
$$

where $\nabla c_{i}\left(x_{i}\right)=2 n_{i}^{2} q_{i} x_{i}+n_{i} b_{i}$. Then, with $x=\left[x_{i}\right]_{i \in V}$, $A=\left[A_{1}, \cdots A_{N}\right], F(x)=\left[\nabla_{i} J_{i}(x)\right]_{i \in V}$ is given by

$F(x)=\nabla c(x)+\operatorname{diag}\left(\left[A_{i}^{T} Z A_{i}\right]_{i \in V}\right) x+A^{T} Z A x-A^{T} \bar{P}$

where $\nabla c(x)=2 \operatorname{diag}\left(\left[n_{i}^{2} q_{i}\right]_{i \in V}\right) x+\left[n_{i} b_{i}\right]_{i \in V}$. Combining terms, $F(x)$ can be written compactly as,

$$
F(x)=Q x+r, \text { where } Q:=\Sigma+A^{T} Z A,
$$

$\Sigma:=\operatorname{diag}\left(\left[2 n_{i}^{2} q_{i}+A_{i}^{T} Z A_{i}\right]_{i \in V}\right)$ andr $:=\left[n_{i} b_{i}\right]_{i \in V}-A^{T} \bar{P}$. Since $q_{i}>0$ and $z_{k}>0, \Sigma \succ 0$ and $A^{T} Z A \succeq 0$, hence $Q \succ 0$. Thus $F(x)$ is Lipschitz continuous with $\theta_{0}=$ $\|Q\|_{2}$ where $\|Q\|_{2}=\sigma_{\max }(Q)$.

Using (21) with $x$ replaced by $x^{i}=\left(x_{i}, x_{-i}^{i}\right)$, yields

$$
\nabla_{i} J_{i}\left(x^{i}\right)=\nabla c_{i}\left(x_{i}\right)+A_{i}^{T} Z A_{i} x_{i}-A_{i}^{T}\left(\bar{P}-Z A x^{i}\right) .
$$

Thus, $\mathbf{F}(\mathbf{x}):=\left[\nabla_{i} J_{i}\left(x^{i}\right)\right]_{i \in V},(16)$, is given by

$$
\begin{aligned}
\mathbf{F}(\mathbf{x}) & =\nabla c(x)+\operatorname{diag}\left(\left[A_{i}^{T} Z A_{i}\right]_{i \in V}\right) x-A^{T} \bar{P} \\
& +\operatorname{diag}\left(\left[A_{i}^{T} Z A\right]_{i \in V}\right) \mathbf{x}
\end{aligned}
$$

Using $\nabla c(x), \Sigma$ and $r$ defined above, and $\mathbf{R}^{T}\left(I_{N} \otimes\right.$ $\left.A^{T} Z A\right)=\operatorname{diag}\left(\left[e_{i}^{T} A^{T} Z A\right]_{i \in V}\right)$ for the last term, yields

$$
\mathbf{F}(\mathbf{x})=\Sigma \mathbf{R}^{T} \mathbf{x}+r+\mathbf{R}^{T}\left(I_{N} \otimes A^{T} Z A\right) \mathbf{x} .
$$

where $x=\mathbb{R}^{T} \mathbf{x}$ was used. Since $\Sigma$ is block-diagonal, $\Sigma \mathbf{R}^{T}=\mathbf{R}^{T}\left(I_{N} \otimes \Sigma\right)$, and with $Q(22)$ we can write

$$
\mathbf{F}(\mathbf{x})=\overline{\mathbf{Q}} \mathbf{x}+r, \quad \text { where } \overline{\mathbf{Q}}:=\mathbf{R}^{T}\left(I_{N} \otimes Q\right) .
$$

Hence, $\|\mathbf{F}(\mathbf{x})-\mathbf{F}(\mathbf{y})\| \leq\|\overline{\mathbf{Q}}\|_{2}\|\mathbf{x}-\mathbf{y}\|$, where $\|\overline{\mathbf{Q}}\|_{2}=$ $\left\|\mathbf{R}^{T}\left(I_{N} \otimes Q\right)\right\|_{2} \leq\left\|\mathbf{R}^{T}\right\|_{2}\left\|I_{N} \otimes Q\right\|_{2}=\|Q\|_{2}$ since $\|\mathbf{R}\|_{2}=1$ and $\left\|I_{N} \otimes Q\right\|_{2}=\|Q\|_{2}$, and thus $\theta=\theta_{0}$.

Remark 3 Note that Assumption 4 on $\mathbf{F}$ is weaker than 
strong monotonicity in Ye and Hu (2017)), or cocoercivty in Salehisadaghiani and Pavel (2017), Shi and Pavel (2017). In ADMM for DOP, cocoercivity of the full gradient is used, which is automatically satisfied by joint convexity of each decoupled $f_{i},(1)$, and Lipschitz continuity of its gradient (see Chang et al. (2015)). In contrast, in a game, because of coupling to the others' actions in $J_{i}$ (2) and because of partial convexity (see Assumption 1), monotonicity of the pseudo-gradient is not automatically satisfied when extended to the augmented space. The next result shows how under Assumption 4, a monotonicity property can be achieved in the augmented space.

Lemma 2 Consider that Assumptions 1, 2, 3 and 4 hold and let

$$
\Psi=\left[\begin{array}{cc}
\frac{\mu}{N} & -\frac{\theta+\theta_{0}}{2 \sqrt{N}} \\
-\frac{\theta+\theta_{0}}{2 \sqrt{N}} & c_{0} \lambda_{2}(L)-\theta
\end{array}\right]
$$

Then, for any $c_{0}>c_{\min }$ where $c_{\min } \lambda_{2}(L)=\frac{\left(\theta+\theta_{0}\right)^{2}}{4 \mu}+\theta$, $\Psi \succ 0$, and for any $\mathbf{x}$ and any $\mathbf{y} \in \operatorname{Ker}(\mathbf{L})$,

$(\mathbf{x}-\mathbf{y})^{T}\left(\mathbf{R F}(\mathbf{x})-\mathbf{R F}(\mathbf{y})+c_{0} \mathbf{L}(\mathbf{x}-\mathbf{y})\right) \geq \bar{\mu}\|\mathbf{x}-\mathbf{y}\|^{2}$,

where $\bar{\mu}:=\lambda_{\min }(\Psi)>0$.

Proof. See Appendix B.

Remark 4 For quadratic games (see Remark 2), $\theta=\theta_{0}=\|Q\|_{2}, F(x)$ is strongly monotone with $\mu=$ $\lambda_{\min }(Q)>0$ (by Assumption 2), hence the $c_{0}$ bound in Lemma 2 simplifies to $c_{0} \lambda_{2}(L)>\frac{\|Q\|_{2}^{2}}{\mu}+\|Q\|_{2}$. Furthermore, if $Q$ is symmetric, $\|Q\|_{2}=\sigma_{\max }(Q)=\lambda_{\max }(Q)$, and this reduces to $c_{0}>\frac{\lambda_{\max }(Q)}{\lambda_{2}(L)}\left(\frac{\lambda_{\max }(Q)}{\lambda_{\min }(Q)}+1\right)$, showing the trade-off between game properties and communication graph connectivity.

Lemma 2 shows that, for a sufficiently large $c_{0}, \mathbf{R F}(\mathbf{x})+$ $c_{0} \mathbf{L x}$ is strongly monotone in a restricted set of directions $\mathbf{x}-\mathbf{y}$, where $\mathbf{y} \in \operatorname{Ker}(\mathbf{L})$. We can call $\mathbf{R F}$ a pre-L-monotone function, related to the concept of premonotone gradient of a prox-regular function, Poliquin and Rockafellar (1996). In fact, being in a restricted set of directions, this is a weaker monotonicity property, similar to the notion of restricted strong convexity used in DOP and high-dimensional statistical estimation Zhang (2017), Agarwal et al. (2010), Negahban et al. (2009). To show this key property, an instrumental step is the decomposition of the augmented space $\mathbb{R}^{N^{2}}$ into the consensus subspace, $\operatorname{Ker}(\mathbf{L})$ (where $\mathbf{F}$ is strongly monotone), and its orthogonal complement, $\operatorname{Ker}(\mathbf{L})^{\perp}$ (where $\mathbf{L}$ is strongly monotone). Based on the Lipschitz continuity of $\mathbf{F}$, for a sufficiently large $c_{0}$, excess strong monotonicity of $c_{0} \mathbf{L}$ can balance the cross-terms (shortage of monotonicity of $\mathbf{R F}$ ) when $\mathbf{x}$ is off the consensus subspace $\operatorname{Ker}(\mathbf{L})$, but not on $\operatorname{Ker}(\mathbf{L})^{\perp}$, (see proof in Appendix B). We show next that this property is sufficient to prove convergence of Algorithm 1.

Theorem 1 Consider that Assumptions 1, 2, 3 and 4 hold. Take an arbitrary $c>0$, any $c_{0}>c_{\min }$, with $c_{\min }$ as in Lemma 2, and let $\bar{c}=c+c_{0}$. If $\beta_{i}>0$ are chosen such that

$$
\lambda_{\min }(\mathbb{B}+2 \bar{c} \mathbb{D}-c L)>\frac{\bar{\theta}^{2}}{2 \bar{\mu}}
$$

where $\mathbb{B}:=\operatorname{diag}\left(\left(\beta_{i}\right)_{i \in V}\right), \mathbb{D}$ and $L$ are the degree and Laplacian matrices of $G_{c}, \bar{\theta}=\theta+2 c_{0} d^{*}, d^{*}$ is the maximal degree of $G_{c}$ and $\bar{\mu}$ is defined in Lemma 2, then the sequence $\left\{x^{i}(k)\right\} \forall i \in V$, or $\{\mathbf{x}(k)\}$, generated by Algorithm 1 converges to $N E$ of game (2) $x^{*}$, (or $\left.\mathbf{1}_{N} \otimes x^{*}\right)$.

Proof. Recall that $\mathbf{G}(\mathbf{x}(\mathbf{k}))=G(x(k))=\left[\partial I_{\Omega_{i}}\left(x_{i}(k)\right)\right]_{i \in V}$. From the $\mathbf{x}$-update (18) in the vector form of Algorithm 1 (see Proposition 1), it follows that there exists $y \in G(x(k)) \subset \mathbb{R}^{N}$ such that,

$$
\begin{aligned}
\mathbf{0}_{N^{2}} & =\mathbf{R}(\mathbf{F}(\mathbf{x}(k-1))+y)+\mathbf{w}(k) \\
& +\left((\mathbb{B}+2 \bar{c} \mathbb{D}) \otimes I_{N}\right)(\mathbf{x}(k)-\mathbf{x}(k-1))+\bar{c} \mathbf{L x}(k-1)
\end{aligned}
$$

or, substituting $\mathbf{w}(k)$ by $\mathbf{w}(k+1)-c \mathbf{L x}(k)$ (cf. (18)),

$$
\begin{aligned}
\mathbf{0}_{N^{2}} & =\mathbf{R} \mathbf{F}(\mathbf{x}(k-1))+\mathbf{R} y+\mathbf{w}(k+1)-c \mathbf{L x}(k) \\
& +\left((\mathbb{B}+2 \bar{c} \mathbb{D}) \otimes I_{N}\right)(\mathbf{x}(k)-\mathbf{x}(k-1))+\bar{c} \mathbf{L} \mathbf{x}(k-1) .
\end{aligned}
$$

Using $\bar{c}=c+c_{0}$ and $H:=\mathbb{B}+2 \bar{c} \mathbb{D}-c L$ this yields

$$
\begin{aligned}
\mathbf{0}_{N^{2}} & =\mathbf{R} \mathbf{F}(\mathbf{x}(k-1))+\mathbf{R} y+\mathbf{w}(k+1) \\
& +\left(H \otimes I_{N}\right)(\mathbf{x}(k)-\mathbf{x}(k-1))+c_{0} \mathbf{L x}(k-1) .
\end{aligned}
$$

Note that $H:=\mathbb{B}+c(2 \mathbb{D}-L)+2 c_{0} \mathbb{D}$ hence $H \otimes I_{N} \succ 0$, since $\mathbb{B} \succ 0$ and $c(2 \mathbb{D}-L) \succeq 0$.

Consider NE $x^{*}$ and let $\mathbf{x}^{*}=\mathbf{1}_{N} \otimes x^{*}$. Then, from (8), with $\mathbf{F}\left(\mathbf{1}_{N} \otimes x^{*}\right)=F\left(x^{*}\right)$, and the definition of $\mathbf{R}$, it follows that $\mathbf{0}_{N^{2}} \in \mathbf{R F}\left(\mathbf{x}^{*}\right)+\mathbf{R G}\left(\mathbf{x}^{*}\right)$, i.e., there exists $y^{*} \in \mathbf{G}\left(\mathbf{x}^{*}\right)$ such that $\mathbf{R} \mathbf{F}\left(\mathbf{x}^{*}\right)+\mathbf{R} y^{*}=\mathbf{0}_{N^{2}}$. Moreover, there exists $\mathbf{q}^{*} \in \mathbb{R}^{N^{2}}$ (any $\mathbf{q}^{*} \in \operatorname{Ker}(\mathbf{L})$ with structure $\mathbf{1}_{N} \otimes \widetilde{q}$, for $\left.\widetilde{q} \in \mathbb{R}^{N}\right)$ such that

$$
\mathbf{0}_{N^{2}}=\mathbf{R} \mathbf{F}\left(\mathbf{x}^{*}\right)+\mathbf{R} y^{*}+c \mathbf{L} \mathbf{q}^{*} \text {. }
$$

Subtracting (28) from (27), multiplying by $\left(\mathbf{x}(k)-\mathbf{x}^{*}\right)^{T}$ and using $\mathbf{R}^{T} \mathbf{x}=x$, yields

$$
\begin{aligned}
& \left(\mathbf{R F}(\mathbf{x}(k-1))-\mathbf{R F}\left(\mathbf{x}^{*}\right)\right)^{T}\left(\mathbf{x}(k)-\mathbf{x}^{*}\right) \\
& +\left(y-y^{*}\right)^{T}\left(x(k)-x^{*}\right) \\
& +\left(\mathbf{w}(k+1)-c \mathbf{L} \mathbf{q}^{*}\right)^{T}\left(\mathbf{x}(k)-\mathbf{x}^{*}\right) \\
& +(\mathbf{x}(k)-\mathbf{x}(k-1))^{T}\left(H \otimes I_{N}\right)\left(\mathbf{x}(k)-\mathbf{x}^{*}\right) \\
& +\mathbf{x}(k-1)^{T} c_{0} \mathbf{L}\left(\mathbf{x}(k)-\mathbf{x}^{*}\right)=0 .
\end{aligned}
$$

Combine the first and fifth term in (29) and write,

$$
\begin{aligned}
& \left(\mathbf{R F}(\mathbf{x}(k-1))+c_{0} \mathbf{L} \mathbf{x}(k-1)-\mathbf{R F}\left(\mathbf{x}^{*}\right)\right)^{T}\left(\mathbf{x}(k)-\mathbf{x}^{*}\right) \\
& =\left(\mathbf{R F}(\mathbf{x}(k-1))-\mathbf{R F}\left(\mathbf{x}^{*}\right)+c_{0} \mathbf{L} \mathbf{x}(k-1)\right)^{T}\left(\mathbf{x}(k-1)-\mathbf{x}^{*}\right) \\
& +\left(\mathbf{R F}(\mathbf{x}(k-1))-\mathbf{R F}\left(\mathbf{x}^{*}\right)+c_{0} \mathbf{L} \mathbf{x}(k-1)\right)^{T}(\mathbf{x}(k)-\mathbf{x}(k-1)) .
\end{aligned}
$$

For the first term on the right-hand side of (30) we use (25) in Lemma 2 (with $\mathbf{L} \mathbf{x}^{*}=\mathbf{0}_{N^{2}}$ ), while for the second term we use (3) for $\rho=\frac{\bar{\theta}^{2}}{2 \bar{\mu}}, \bar{\theta}=\theta+2 c_{0} d^{*}$. This yields,

$\left(\mathbf{R F}(\mathbf{x}(k-1))-\mathbf{R F}\left(\mathbf{x}^{*}\right)+c_{0} \mathbf{L x}(k-1)\right)^{T}\left(\mathbf{x}(k)-\mathbf{x}^{*}\right)$ 


$$
\begin{aligned}
& \left.\geq \bar{\mu} \| \mathbf{x}(k-1)-\mathbf{x}^{*}\right) \|^{2} \\
& -\frac{\bar{\mu}}{\overline{\theta^{2}}}\left\|\mathbf{R F}(\mathbf{x}(k-1))-\mathbf{R F}\left(\mathbf{x}^{*}\right)+c_{0} \mathbf{L x}(k-1)\right\|^{2} \\
& -\frac{\bar{\theta}^{2}}{4 \bar{\mu}}\|\mathbf{x}(k)-\mathbf{x}(k-1)\|^{2}
\end{aligned}
$$

Note that $\mathbf{L}$ is $\|\mathbf{L}\|_{2}$-Lipschitz with $\|\mathbf{L}\|_{2}=\lambda_{\max }(L) \leq$ $2 d^{*}$. Based on this, with $\mathbf{L} \mathbf{x}^{*}=\mathbf{0}_{N^{2}}$, Assumption 4 for $\mathbf{F},\|\mathbf{R}\|_{2}=1$ and the triangle inequality, we can write for the second term on the right-hand side of (31),

$\left.\left\|\mathbf{R F}(\mathbf{x}(k-1))-\mathbf{R F}\left(\mathbf{x}^{*}\right)+c_{0} \mathbf{L x}(k-1)\right\| \leq \bar{\theta} \| \mathbf{x}(k-1)-\mathbf{x}^{*}\right) \|$

where $\bar{\theta}=\theta+2 c_{0} d^{*}$. Using this in (31) yields

$$
\begin{aligned}
& \left(\mathbf{R F}(\mathbf{x}(k-1))-\mathbf{R F}\left(\mathbf{x}^{*}\right)+c_{0} \mathbf{L x}(k-1)\right)^{T}\left(\mathbf{x}(k)-\mathbf{x}^{*}\right) \\
& \geq-\frac{\bar{\theta}^{2}}{4 \bar{\mu}}\|\mathbf{x}(k)-\mathbf{x}(k-1)\|^{2}
\end{aligned}
$$

Back to (29), for the second term, since $y \in G(x(k))$, $y^{*} \in G\left(x^{*}\right), \mathcal{I}_{\Omega_{i}}$ is convex (Assumption 1) it follows that

$$
\left(y-y^{*}\right)^{T}\left(x(k)-x^{*}\right) \geq 0 .
$$

Finally for the third term in (29), we use the following. From (18) with zero initial conditions, it follows that $\mathbf{w}(k+1)=c \mathbf{L q}(k)$, where $\mathbf{q}(k)=\sum_{t=0}^{k} \mathbf{x}(t)$. Thus,

$$
\mathbf{q}(k)-\mathbf{q}(k-1)=\mathbf{x}(k)
$$

Then, we can write

$$
\begin{aligned}
& \left(\mathbf{w}(k+1)-c \mathbf{L} \mathbf{q}^{*}\right)^{T}\left(\mathbf{x}(k)-\mathbf{x}^{*}\right) \\
& =\left(\mathbf{q}(k)-\mathbf{q}^{*}\right)^{T} c \mathbf{L}\left(\mathbf{q}(k)-\mathbf{q}(k-1)-\mathbf{x}^{*}\right) \\
& =\left(\mathbf{q}(k)-\mathbf{q}^{*}\right)^{T} c \mathbf{L}(\mathbf{q}(k)-\mathbf{q}(k-1))
\end{aligned}
$$

Using (32), (33) and this last relation in (29) yields

$$
\begin{aligned}
& -\frac{\bar{\theta}^{2}}{4 \bar{\mu}}\|\mathbf{x}(k)-\mathbf{x}(k-1)\|^{2}+\left(\mathbf{q}(k)-\mathbf{q}^{*}\right)^{T} c \mathbf{L}(\mathbf{q}(k)-\mathbf{q}(k-1)) \\
& +(\mathbf{x}(k)-\mathbf{x}(k-1))^{T}\left(H \otimes I_{N}\right)\left(\mathbf{x}(k)-\mathbf{x}^{*}\right) \leq 0,
\end{aligned}
$$

where $H \otimes I_{N} \succ 0$. Using (4) to deal with all cross-terms in the previous inequality, yields

$$
\begin{array}{r}
\left\|\mathbf{x}(k)-\mathbf{x}^{*}\right\|_{H \otimes I_{N}}^{2}+\left\|\mathbf{q}(k)-\mathbf{q}^{*}\right\|_{c \mathbf{L}}^{2}-\left\|\mathbf{x}(k-1)-\mathbf{x}^{*}\right\|_{H \otimes I_{N}}^{2} \\
-\left\|\mathbf{q}(k-1)-\mathbf{q}^{*}\right\|_{c \mathbf{L}}^{2} \leq-\|\mathbf{x}(k)-\mathbf{x}(k-1)\|_{H \otimes I_{N}}^{2} \\
-\|\mathbf{q}(k)-\mathbf{q}(k-1)\|_{c \mathbf{L}}^{2}+\frac{\bar{\theta}^{2}}{2 \bar{\mu}}\|\mathbf{x}(k)-\mathbf{x}(k-1)\|^{2} .
\end{array}
$$

Let us make the following notations:

$$
\mathbf{z}(k):=\left[\begin{array}{l}
\mathbf{x}(k) \\
\mathbf{q}(k)
\end{array}\right], \mathbf{z}^{*}:=\left[\begin{array}{l}
\mathbf{x}^{*} \\
\mathbf{q}^{*}
\end{array}\right], \Phi=\left[\begin{array}{ll}
H & \mathbf{0}_{N \times N} \\
\mathbf{0}_{N \times N} & c L
\end{array}\right]
$$

Then we can write the last inequality as $\left\|\mathbf{z}(k)-\mathbf{z}^{*}\right\|_{\Phi \otimes I_{N}}^{2}-\left\|\mathbf{z}(k-1)-\mathbf{z}^{*}\right\|_{\Phi \otimes I_{N}}^{2}$
$\leq-\|\mathbf{z}(k)-\mathbf{z}(k-1)\|_{\Phi \otimes I_{N}}^{2}+\frac{\bar{\theta}^{2}}{2 \bar{\mu}}\|\mathbf{x}(k)-\mathbf{x}(k-1)\|^{2}$.

Moreover, since $\frac{\bar{\theta}^{2}}{2 \bar{\mu}}\|\mathbf{x}(k)-\mathbf{x}(k-1)\|^{2} \leq \frac{\bar{\theta}^{2}}{2 \bar{\mu} \lambda_{\min }(H)} \| \mathbf{x}(k)-$ $\mathbf{x}(k-1)\left\|_{H \otimes I_{N}}^{2} \leq \frac{\bar{\theta}^{2}}{2 \bar{\mu} \lambda_{\min }(H)}\right\| \mathbf{z}(k)-\mathbf{z}(k-1) \|_{\Phi \otimes I_{N}}^{2}$, from the foregoing it follows that

$$
\begin{array}{r}
\left\|\mathbf{z}(k)-\mathbf{z}^{*}\right\|_{\Phi \otimes I_{N}}^{2}-\left\|\mathbf{z}(k-1)-\mathbf{z}^{*}\right\|_{\Phi \otimes I_{N}}^{2} \leq \\
-\zeta\|\mathbf{z}(k)-\mathbf{z}(k-1)\|_{\Phi \otimes I_{N}}^{2} \leq 0
\end{array}
$$

where $\zeta=1-\frac{\bar{\theta}^{2}}{2 \bar{\mu} \lambda_{\min }(H)}$, and $0<\zeta<1$ by (26). Summing (37) over $k$ from 1 to $\infty$ yields

$\sum_{k=1}^{\infty}\|\mathbf{z}(k)-\mathbf{z}(k-1)\|_{\Phi \otimes I_{N}}^{2} \leq \frac{1}{\zeta}\left\|\mathbf{z}(0)-\mathbf{z}^{*}\right\|_{\Phi \otimes I_{N}}^{2}<\infty(38)$

From (38) it follows that $\|\mathbf{z}(k)-\mathbf{z}(k-1)\|_{\Phi \otimes I_{N}}^{2} \rightarrow 0$. From (37), since $\left\|\mathbf{z}(k)-\mathbf{z}^{*}\right\|_{\Phi \otimes I_{N}}^{2}$ or $\left\|\mathbf{x}(k)-\mathbf{x}^{*}\right\|_{H \otimes I_{N}}^{2}+$ $\left\|\mathbf{q}(k)-\mathbf{q}^{*}\right\|_{c \mathbf{L}}^{2}$ is bounded and non-increasing, it follows that $\left\|\mathbf{x}(k)-\mathbf{x}^{*}\right\|_{H \otimes I_{N}}^{2}+\left\|\mathbf{q}(k)-\mathbf{q}^{*}\right\|_{c \mathbf{L}}^{2} \rightarrow v$, for some $v \geq 0$, where $\left(H \otimes I_{N}\right) \succ 0, \mathbf{L} \succeq 0$.

Any $\mathbf{q} \in \mathbb{R}^{N^{2}}$ can be decomposed as $\mathbf{q}=\mathbf{q}^{\|}+\mathbf{q}^{\perp}$, where $\mathbf{q}^{\|} \in \operatorname{Ker}(\mathbf{L}), \mathbf{q}^{\perp} \in \operatorname{Ker}(\mathbf{L})^{\perp}$, with $\left(\mathbf{q}^{\perp}\right)^{T} \mathbf{L} \mathbf{q}^{\perp}>0$, $\forall \mathbf{q}^{\perp} \neq 0$. Decomposing $\mathbf{q}(k)$ and $\mathbf{q}^{*}$ into $(\cdot)^{\|}$and $(\cdot)^{\perp}$ components, $\left\|\mathbf{q}(k)-\mathbf{q}^{*}\right\|_{c \mathbf{L}}^{2}=\left\|\mathbf{q}(k)^{\perp}-\mathbf{q}^{* \perp}\right\|_{c \mathbf{L}}^{2}$, with $\mathbf{q}(k)^{\perp}, \mathbf{q}^{* \perp} \in \operatorname{Ker}(\mathbf{L})^{\perp}$. Hence, from the above,

$$
V_{x^{*}}(k):=\left\|\mathbf{x}(k)-\mathbf{x}^{*}\right\|_{H \otimes I_{N}}^{2}+\left\|\mathbf{q}(k)^{\perp}-\mathbf{q}^{* \perp}\right\|_{c \mathbf{L}}^{2}
$$

is bounded and non-increasing and converges to $v$. Thus, since $H \succ 0$ and $\left\|\mathbf{q}(k)^{\perp}-\mathbf{q}^{* \perp}\right\|_{c \mathbf{L}}^{2}>0, \forall \mathbf{q}^{\perp}(k) \neq 0$, the sequence $\left[\mathbf{x}(k) ; \mathbf{q}(k)^{\perp}\right]$ is bounded, hence has at least a limit point $\left[\overline{\mathbf{x}} ; \overline{\mathbf{q}}^{\perp}\right]$, where $\overline{\mathbf{q}}^{\perp} \in \operatorname{Ker}(\mathbf{L})^{\perp}$. Also, w $(k+$ 1) $=c \mathbf{L q}(k)=c \mathbf{L} \mathbf{q}(k)^{\perp}$, has a limit point $\overline{\mathbf{w}}=c \mathbf{L} \overline{\mathbf{q}}^{\perp}$. By Lemma 1, any limit point of $\mathbf{x}(k)$ satisfies $\overline{\mathbf{x}}=\mathbf{1}_{N} \otimes$ $x^{*}$, with $x^{*}$ NE of the game and $\overline{\mathbf{w}}$ such that (19) holds, hence $\overline{\mathbf{x}}=\mathbf{x}^{*}$. Consider now

$$
V_{\bar{x}}(k):=\left\|\mathbf{x}(k)-\mathbf{x}^{*}\right\|_{H \otimes I_{N}}^{2}+\left\|\mathbf{q}(k)^{\perp}-\overline{\mathbf{q}}^{\perp}\right\|_{c \mathbf{L}}^{2}
$$

Similar to the first part of the proof for $V_{x^{*}}(k)$ with $\mathbf{x}^{*}$ $c \mathbf{L} \mathbf{q}^{*}$ and (28), it can be shown that $V_{\bar{x}}(k)$ is bounded and non-increasing, by using (19) for $\overline{\mathbf{x}}=\mathbf{x}^{*}, \overline{\mathbf{w}}=c \mathbf{L} \overline{\mathbf{q}}^{\perp}$ instead of (28). Hence, $V_{\bar{x}}(k)$ converges to some $\bar{v} \geq 0$ as $k \rightarrow \infty$. This $\bar{v}$ is the same along any subsequence. Since $\left[\mathbf{x}^{*} ; \overline{\mathbf{q}}^{\perp}\right]$ is a limit point for $\left[\mathbf{x}(k) ; \mathbf{q}(k)^{\perp}\right]$, there exists a subsequence $\left\{k_{n}\right\}, k_{n} \rightarrow \infty$ as $n \rightarrow \infty$, such that $\left\{\left[\mathbf{x}\left(k_{n}\right) ; \mathbf{q}\left(k_{n}\right)^{\perp}\right]\right\} \rightarrow\left[\mathbf{x}^{*} ; \overline{\mathbf{q}}^{\perp}\right]$ as $n \rightarrow \infty$. Taking limit of $V_{\bar{x}}\left(k_{n}\right),(39)$, along this $\left\{k_{n}\right\}$ subsequence, as $n \rightarrow \infty$, it follows that $\bar{v}=0$. Since $\left(H \otimes I_{N}\right) \succ 0$ and $\overline{\mathbf{q}}^{\perp}, \mathbf{q}(k)^{\perp} \in \operatorname{Ker}(\mathbf{L})^{\perp}$, taking limit in (39) as $k \rightarrow \infty$, it follows that $\mathbf{x}(k) \rightarrow \mathbf{x}^{*}$, with $\mathbf{x}^{*}=\mathbf{1}_{N} \otimes x^{*}$ and $x^{*} \mathrm{NE}$.

Remark 5 We note that, unlike ADMM for DOP, Wei and Ozdaglar (2013), Hong et al. (2016), to prove convergence we cannot use a common objective function 
and common Lagrangian. Instead our proof relies on properties of the extended pseudo-gradient $\mathbf{F}$. Compared to Salehisadaghiani and Pavel (2017), Shi and Pavel (2017), where $c_{0}=0$ and cocoercivity of $\mathbf{R F}$ was assumed, here we only assume Lipschitz continuity of $\mathbf{F}$. We achieved this relaxation by slightly modifying the ADMM algorithm so that the $\mathbf{x}$-update uses the extra $c_{0}>$ 0 parameter in the proximal-approximation (quadratic penalty). Convergence is proved for sufficiently large $c_{0}$. This is similar to the way in which non-convexity is overcome in ADMM for DOP in Hong et al. (2016), Wang et al. (2015)). Based on Gershgorin theorem, a sufficient diagonal-dominance condition can be derived for (26) to hold, and $\beta_{i}$ parameters can be selected independently by players (see e.g. Yi and Pavel (2017)). We note that assumptions on $F$ and $\mathbf{F}$ could be relaxed to hold only locally around $x^{*}$ and $\mathbf{x}^{*}$, in which case all results become local. We also note that the class of quadratic games satisfies all asumptions globally.

\subsection{Convergence Rate Analysis}

Next we investigate the convergence rate of Algorithm 1. We use the following result.

Proposition 2 (Shi et al. (2015)) If a sequence $\{a(k)\} \subset \mathbb{R}$ is: (i) nonnegative, $a(k) \geq 0$, (ii) summable, $\sum_{k=1}^{\infty} a(k)<\infty$, and (iii) monotonically non-increasing, $a(k+1) \leq a(k)$, then we have: $a(k)=o\left(\frac{1}{k}\right)$, i.e., $\lim _{k \rightarrow \infty} k a(k)=0$.

For $\|\mathbf{z}(k-1)-\mathbf{z}(k)\|_{\Phi \otimes I_{N}}^{2}$, with $\mathbf{z}(k), \Phi \otimes I_{N}$ as in (36), (36), summability follows from (38) in Theorem 1 . Next we provide a lemma showing that $\|\mathbf{z}(k-1)-\mathbf{z}(k)\|_{\Phi \otimes I_{N}}^{2}$ is monotonically non-increasing.

Lemma 3 Under the assumptions of Theorem 1, the sequence $\{\mathbf{z}(k)\},(36)$, generated by Algorithm 1 satisfies for all $k \geq 1$,

$$
\|\mathbf{z}(k)-\mathbf{z}(k+1)\|_{\Phi \otimes I_{N}}^{2} \leq\|\mathbf{z}(k-1)-\mathbf{z}(k)\|_{\Phi \otimes I_{N}}^{2},
$$

where $\Phi \otimes I_{N} \succeq 0$ is as in (36).

Proof. Let $\Delta \mathbf{x}(k+1):=\mathbf{x}(k)-\mathbf{x}(k+1), \Delta \mathbf{q}(k+1):=$ $\mathbf{q}(k)-\mathbf{q}(k+1), \Delta \mathbf{z}(k+1):=\mathbf{z}(k)-\mathbf{z}(k+1), \Delta \mathbf{F}(\mathbf{x}(k+$ 1)) $:=\mathbf{F}(\mathbf{x}(k))-\mathbf{F}(\mathbf{x}(k+1)), \Delta \mathbf{G}(x(k+1))=\mathbf{G}(x(k))-$ $\mathbf{G}(x(k+1))$. Following similar arguments as in the proof of Theorem 1, we obtain similar to (37), $\|\Delta \mathbf{z}(k)\|_{\Phi \otimes I_{N}}^{2}$ $\|\Delta \mathbf{z}(k+1)\|_{\Phi \otimes I_{N}}^{2} \geq \zeta\|\Delta \mathbf{z}(k)-\Delta \mathbf{z}(k+1)\|_{\Phi \otimes I_{N}}^{2} \geq 0$, where $\zeta=1-\frac{\bar{\theta}^{2}}{2 \bar{\mu} \lambda_{\min }(H)}>0$, by $(26)$.

Theorem 2 Under the same assumptions of Theorem 1, the following rates hold for Algorithm 1:

$$
\left\|\mathbf{x}^{\perp}(k)\right\|_{\mathbf{L}}^{2}=o\left(\frac{1}{k}\right),
$$

where $\mathbf{x}^{\perp}(k) \in \operatorname{Ker}(\mathbf{L})^{\perp}, \mathbf{L}=L \otimes I_{N}, L$ the Laplacian matrix of $G_{c}$, and for some $y \in \mathbf{G}(x(k))$,

$\left\|\mathbf{R}(\mathbf{F}(\mathbf{x}(k-1))+y)+\mathbf{w}(k+1)+c_{0} \mathbf{L} \mathbf{x}^{\perp}(k-1)\right\|_{\left(H \otimes I_{N}\right)^{-1}}^{2}=o\left(\frac{1}{k}\right)$
Proof. By Theorem 1 (equation (38)), $\sum_{k=0}^{\infty} \| \mathbf{z}(k-1)-$ $\mathbf{z}(k) \|_{\Phi \otimes I_{N}}^{2}<\infty$. Moreover, Lemma 3 proves that $\| \mathbf{z}(k-$ 1) $-\mathbf{z}(k) \|_{\Phi \otimes I_{N}}^{2}$ is monotonically non-increasing. Then, it directly follows by Proposition 2 that,

$$
\|\mathbf{z}(k-1)-\mathbf{z}(k)\|_{\Phi \otimes I_{N}}^{2}=o\left(\frac{1}{k}\right) .
$$

Expanding this by using (36) yields,

$\|\mathbf{x}(k-1)-\mathbf{x}(k)\|_{H \otimes I_{N}}^{2}+c\|\mathbf{q}(k-1)-\mathbf{q}(k)\|_{\mathbf{L}}^{2}=o\left(\frac{1}{k}\right)$ and, using (34) $\mathbf{q}(k-1)-\mathbf{q}(k)=\mathbf{x}(k)$, so that

$$
\|\mathbf{x}(k-1)-\mathbf{x}(k)\|_{H \otimes I_{N}}^{2}+c\|\mathbf{x}(k)\|_{\mathbf{L}}^{2}=o\left(\frac{1}{k}\right)
$$

As in the proof of Theorem 1, we can write this as

$$
\|\mathbf{x}(k-1)-\mathbf{x}(k)\|_{H \otimes I_{N}}^{2}+c\left\|\mathbf{x}^{\perp}(k)\right\|_{\mathbf{L}}^{2}=o\left(\frac{1}{k}\right)
$$

where $\mathbf{x}^{\perp}(k) \in \operatorname{Ker}(\mathbf{L})^{\perp}, \mathbf{x}(k)^{\perp} \mathbf{L} \mathbf{x}^{\perp}(k)>0$, for all $\mathbf{x}^{\perp}(k) \neq 0$. If the sum of two positive sequences is $o\left(\frac{1}{k}\right)$, then each sequence is $o\left(\frac{1}{k}\right)$, hence, by the foregoing,

$$
\left\|\mathbf{x}^{\perp}(k)\right\|_{\mathbf{L}}^{2}=o\left(\frac{1}{k}\right) \text {. }
$$

From (40), (27), we can write $\|\mathbf{x}(k)-\mathbf{x}(k-1)\|_{H \otimes I_{N}}^{2}=$ $\left\|\mathbf{R F}(\mathbf{x}(k-1))+\mathbf{R} y+\mathbf{w}(k+1)+c_{0} \mathbf{L} \mathbf{x}^{\perp}(k-1)\right\|_{\left(H \otimes I_{N}\right)^{-1}}^{2}=$ $o\left(\frac{1}{k}\right)$, for some $y \in \mathbf{G}(x(k))$.

Remark 6 Since $\mathbf{x}^{\perp}(k) \in \operatorname{Ker}(\mathbf{L})^{\perp},\left\|\mathbf{x}^{\perp}(k)\right\|_{\mathbf{L}}^{2}$ can be interpreted as a weighted distance from the consensus subspace, while the second rate can be interpreted a weighted distance from the optimality conditions. Unlike DOP, e.g. Wei and Ozdaglar (2013), in a game we do not have a common merit function for all players and the convergence rate we are able to show is only in terms of optimality residuals.

\section{Simulation Results}

In this section, we present two examples to illustrate the performance of Algorithm 1. The first one is a networked Nash-Cournot game (Example 1) as in Koshal et al. (2016), while the second one is a rate control game in Wireless Ad-Hoc Network (WANET), as in Salehisadaghiani and Pavel (2018); Alpcan and Başar (2005).

\subsection{Example 1: Nash-Cournot game over networks}

In this section, we examine the performance of the proposed algorithm on a networked Nash-Cournot game (Example 1). We consider $N=20$ firms and $m=7$ markets, (Fig. 1). Each firm $i$ has a local normalized constraint as $0 \leq x_{T, i} \leq 1$ on its total production $x_{i, T}=n_{i} x_{i}$, where $n_{i}$ is the number of markets it participates in, hence $\Omega_{i}=\left[0,1 / n_{i}\right]$. The local objective function is taken as (20), where the local production cost function of firm $i$ is $c_{i}\left(x_{i}\right)=n_{i}{ }^{2} q_{i} x_{i}^{2}+n_{i} b_{i} x_{i}$ where 


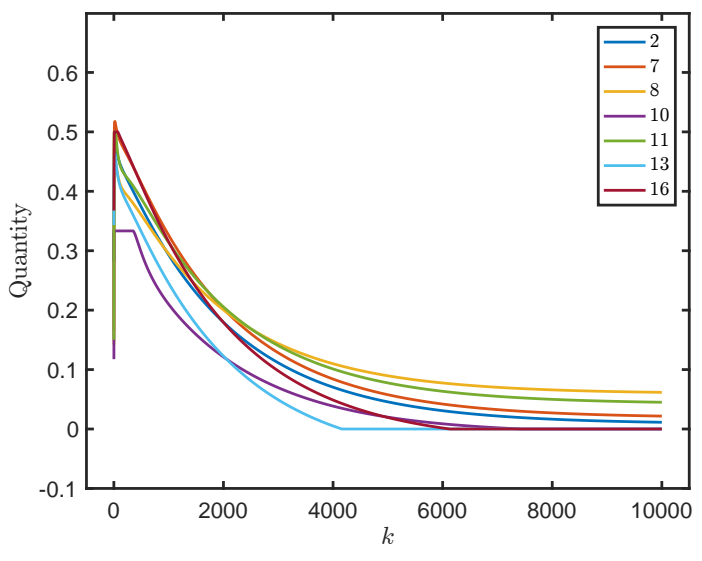

Figure 3. NE computation of a Nash-Cournot game by Algorithm 1 over $G_{c}$ in Fig. 2. The figure shows the convergence paths of 7 randomly selected firms.

$q_{i}, b_{i}$ are randomly drawn from the uniform distribution $U(1,2)$. All assumptions hold globally. In the first case, each local cost function $J_{i}$ is normalized by dividing by $n_{i}^{2} q_{i}$, which yields a better condition number. The price function is taken as the linear function $P=$ $\bar{P}-Z A x$, where $z_{k}=0.01$ and $\bar{P}_{k}$ are randomly drawn from $Z A \Omega_{i}+U(1,2)$. This yields $\lambda_{\min }(Q)=1.001=\mu$, $\lambda_{\max }(Q)=1.099=\theta$. For a communication graph $G_{c}$ as in Fig. 2, $\lambda_{2}(L)=0.102$, and the bound for $c_{0}$ in Lemma 2 is 22.5 . We set $c_{0}=22.5$, an arbitrary step-size $c=1$, and $\beta_{i}=10$ for all firms, satisfying (26). The results for the implementation of Algorithm 1 in the partial decision setting, where the primal variables $x^{i}$ are exchanged over the sparsely connected graph $G_{c}$ (Fig. 2), are shown in Fig. 3. Initial conditions are randomly selected, for $x_{i}$ from $U(0,0.5)$, and for $x_{j}^{i}(i \neq j)$ from $U(0,1)$. The results indicate fast convergence.

Next, we study an extreme case where the costs are widely different. This yields a high condition number, and we can study how the cost functions affect the outcome of market competition. The $q_{i}$ and $b_{i}$ for the first 10 firms are set at the level of 0.01 while those for the other firms are at the level of 100, and each firm $i$ has a local constraint $0 \leq x_{i, T} \leq 100$. Intuitively, this will lead to high/low market occupation of the first/last 10 firms. The price function is taken as the linear function $P=\bar{P}-Z A x$, where $z_{k}$ are randomly drawn from $U(1,10)$ and $\bar{P}_{k}$ randomly generated such that $P_{k}>0$ for all feasible $x$. This yields $\lambda_{\min }(Q)=1.482=\mu$, $\lambda_{\max }(Q)=1005=\theta$, and the bound for $c_{0}$ in Lemma 2 is $6 e 6$. However, we found this bound conservative in our experiments. We plot the sequences of relative error (vs. the 'true' NE obtained by (centralized) gradient method) under different parameter settings in the top sub-figure of Fig. 4. The trends reflected in the top sub-figure of Fig. 4 match the intuition of our algorithm design (see Appendix A) and the implication of (26) in Theorem 1. In general, smaller penalty parameters $c, c_{0}$, and $\beta_{i}$ 's lead to faster convergence but they have to be
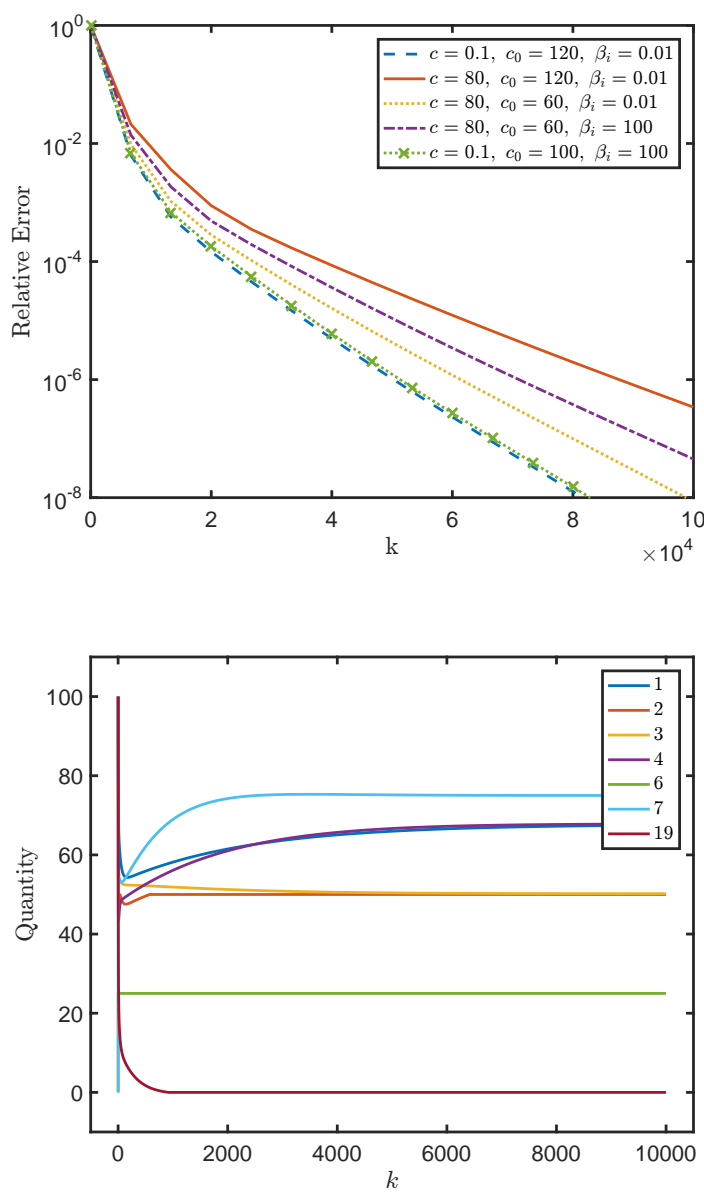

Figure 4. NE computation of a Nash-Cournot game by Algorithm 1 over $G_{c}$ in Fig. 2. The top figure plots relative error $\frac{\left\|x(k)-x^{*}\right\|}{\left\|x(0)-x^{*}\right\|}$ vs. iteration number $k$. The bottom figure shows the convergence paths of 7 selected firms.

large enough to guarantee convergence. An instance of the first $10^{4}$ iterations of trajectories of selected firms are plotted in the bottom sub-figure of Fig. 4 . The initial estimate for $x_{-i}^{i}$ 's are randomly drawn from $(0,200)$; to observe diverse firm trajectories, the first 5 and last 5 firms' local $x_{i}^{i}$ 's are initialized as the upper limits of their local constraints, respectively, while the other firms start from 0 . This way, we can see four cases: (1-5) "aggressive start, rich end"; (6-10) "conservative start, rich end"; (11-15) "conservative start, poor end" (16-20) "aggressive start, poor end".

\subsection{Example 2: Rate control game over WANET}

In this section we consider an engineering networkprotocol example for a rate control game over a Wireless Ad-Hoc Network (WANET), Alpcan and Basar (2002); Alpcan and Başar (2005). Consider 16 mobile nodes interconnected by multi-hop communication paths in a WANET Fig. 5 (a). $N=15$ users/players want to transfer data from a source node to a destination node via this WANET. In Fig. 5 (a) solid lines represent physical links and dashed lines display unique paths that are 


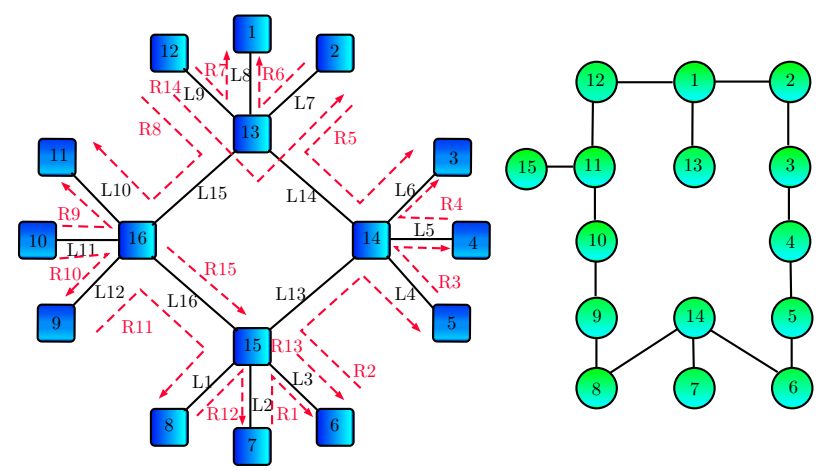

Figure 5. (a) Wireless Ad-Hoc Network (left). (b) Communication graph $G_{c}$ (right) for Example 2.

assigned to each user to transfer its data. Each link has a positive capacity that restricts the users' data flow. In this WANET scenario there is no central node. Each user/player is prescribed to communicate locally with its neighbours over a preassigned (sparse but connected) communication graph $G_{c}$, e.g. as depicted in Fig. 5 (b). In Fig. 5 (b) a node represents a player, and an edge in the graph signifies the ability for the two players to exchange information. Each user exchanges information locally (thus mitigating the lack of centralized information), so that he can estimate the other flows and, based on them, adjust its own decision which is his data rate. Here is the list of WANET notations:

(1) $L_{j}:$ Link $j, j=1, \ldots, 16$,

(2) $R_{i}$ : The path assigned to user $i, i=1, \ldots, 15$,

(3) $C_{j}>0$ : Link $j$ 's capacity, $j=1, \ldots, 16$,

(4) $0 \leq x_{i} \leq 10$ : The data flow of user $i, i=1, \ldots, 15$.

Each path consists of a set of links, e.g., $R_{1}=\left\{L_{2}, L_{3}\right\}$. Each user $i$ decision (action) is its data rate flow $0 \leq$ $x_{i} \leq 10$ to send over $R_{i}$, based on its cost function $J_{i}$,

$J_{i}\left(x_{i}, x_{-i}\right):=\sum_{j: L_{j} \in R_{i}} \frac{\kappa}{C_{j}-\sum_{w: L_{j} \in R_{w}} x_{w}}-\chi_{i} \log \left(x_{i}+1\right)$,

which depends on the flows of the other users, and where $\kappa>0$ and $\chi_{i}>0$ are network-wide known and userspecific parameters, respectively. In this case $\lambda_{2}(L)=$ 0.18 and the other assumptions hold locally. For $\chi_{i}=10$ $\forall i=1, \ldots, 15$ and $C_{j}=10 \forall j=1, \ldots, 16$ we compute numerically $\|D F(x)\|_{2}$ and $\|D \mathbf{F}(x)\|_{2}$ over $\Omega, \theta_{0}=4.7$, $\theta=6.7$. The bound for $c_{0}$ in Lemma 2 is 30.7 . We set $c_{0}=31$, hence $\bar{\mu}=0.04$, an arbitrary step-size $c=1$ and $\beta_{i}=14, \forall i=1, \ldots, 15$ (by (26) in Theorem 1 ). We compare our Algorithm 1 with the gossip-based one proposed in Salehisadaghiani and Pavel (2018) run over $G_{c}$, with diminishing-step sizes. The results are shown in Fig. 6, for uniform randomly selected initial conditions. The simulation results show that Algorithm 1 is about two orders of magnitude faster than the one in Salehisadaghiani and Pavel (2018).

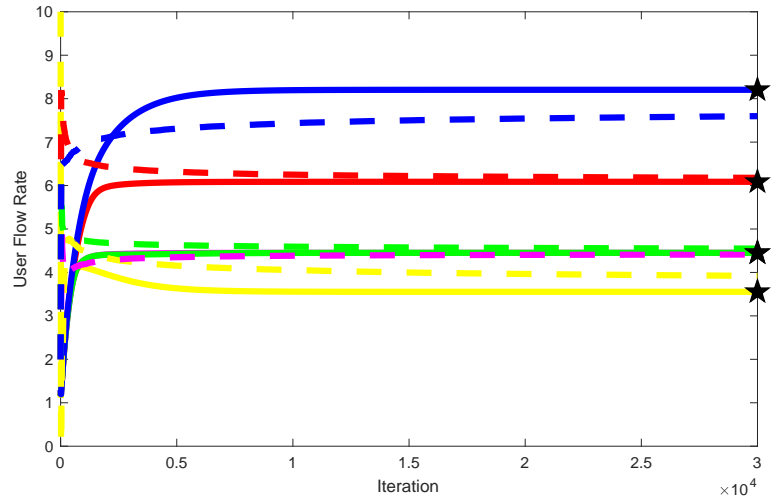

Figure 6. Flow rates of users 1, 3, 5, 8, 13 using Algorithm 1 (solid lines) vs. algorithm in Salehisadaghiani and Pavel (2018) (dashed lines). NE represented by black stars.

\section{Conclusions}

We designed a distributed NE seeking algorithm in general games by using an inexact-ADMM approach. Each player maintains action estimates for the others, exchanged with its neighbours over a connected, undirected communication graph. The game was reformulated as a modified game with virtual consensus constraints for the action estimates, and solved within the framework of inexact-ADMM. An inexact-ADMM algorithm was designed which was shown to convergence to the NE of the game under strong monotonicity of the pseudo-gradient and Lipschitz continuity of the extended pseudo-gradient. The convergence rate of the algorithm was compared with that of an existing gossipbased NE seeking algorithm. To deal with the merely monotone case (thus with possibly multiple NEs) we leave for future work; this would require more involved computations even on the consensus subspace. As other directions for future work we can mention: (1) extension to directed communication graphs, (2) adaptation to games with networked structure (where computation and memory could be reduced if each player maintains only its own required estimates), (3) designing incentives to ensure that players truthfully share their states and auxiliary variables.

\section{A Derivation of ADMM Algorithm 1}

In the following we show how Algorithm 1 can be derived based on an ADMM approach adapted for a game setup. Using slack variables $t^{i j}, t^{j i}$ associated with each edge to separate the constraints and $\mathcal{I}_{\Omega_{i}}\left(x_{i}^{i}\right)$ for the feasibility constraint $x_{i}^{i} \in \Omega_{i}$, we write (10) as

$$
\left\{\begin{aligned}
\underset{x_{i}^{i} \in \mathbb{R}}{\operatorname{minimize}} & J_{i}\left(x_{i}^{i}, x_{-i}^{i}\right)+\mathcal{I}_{\Omega_{i}}\left(x_{i}^{i}\right), \\
\text { subject to } & x^{i}=t^{i j} ; \forall j \text { s.t. }(i, j) \in E \\
& x^{i}=t^{j i} ; \forall j \text { s.t. }(j, i) \in E
\end{aligned}\right.
$$

and similarly for player $j$. We cannot directly use a standard ADMM algorithm because each minimization in 
(A.1) is not over the whole $x^{i}$ as in DOPT, cf. (1), but rather only over part of it (action $x_{i}^{i}$ ), and inherent coupling to the others' decisions still exists indirectly, via the estimates $x_{-i}^{i}$. Note that in (A.1), since minimization of $J_{i}$ is only over the action component $x_{i}^{i}$, for the other (estimate) components $x_{-i}^{i}$ we can consider a zero objective function. We will develop an ADMM-type algorithm to solve (A.1) in a distributed manner. Let $\left\{u^{i j}, v^{j i}\right\} \in \mathbb{R}^{N}$ be the Lagrange multipliers of player $i$, associated with the constraints in (A.1) (source, destination), and similarly, $\left\{u^{j i}, v^{i j}\right\} \in \mathbb{R}^{N}$, for player $j$, respectively. Let $N_{i}^{\text {out }}=\{j \mid(i, j) \in E\}$ and $N_{i}^{i n}=\{j \mid(j, i) \in E\}$, and since $G_{c}$ is undirected, $N_{i}^{\text {in }}=N_{i}^{\text {out }}=N_{i}$. We show next how the algorithm can be derived and simplified so that each player maintains only its dual variables $w^{i}:=\sum_{j \in N_{i}} u^{i j}+v^{j i}$, not all its Lagrangian multipliers $\left\{u^{i j}, v^{j i}\right\}$. The auxiliary variables $t^{i j}, t^{j i}$ are obtained based on minimizing the overall augmented Lagrangian,

$$
\begin{aligned}
& \left.\mathcal{L}^{c}\left(\left\{x^{i}\right\},\left\{t^{i j}, t^{j i}\right\}\right\} ;\left\{u^{i j}, v^{j i}\right\} ;\left\{u^{j i}, v^{i j}\right\}\right):=\sum_{i \in V} J_{i}\left(x_{i}^{i}, x_{-i}^{i}\right) \\
& +\sum_{i \in V} \sum_{j \in N_{i}} u^{i j}\left(x^{i}-t^{i j}\right)+\sum_{i \in V} \sum_{j \in N_{i}} v^{j i^{T}}\left(x^{i}-t^{j i}\right) \quad(\mathrm{A} .2) \\
& +\frac{c}{2} \sum_{i \in V} \sum_{j \in N_{i}}\left\|x^{i}-t^{i j}\right\|^{2}+\frac{c}{2} \sum_{i \in V} \sum_{j \in N_{i}}\left\|x^{i}-t^{j i}\right\|^{2}
\end{aligned}
$$

Based on (A.2), $t^{i j}, t^{j i}$ are updated as:

$$
\begin{aligned}
& t^{i j}(k)=\arg \min _{t^{i j}} \mathcal{L}^{c}\left(\left\{x^{i}(k-1)\right\},\left\{t^{i j}\right\} ;\left\{u^{i j}(k-1), v^{i j}(k-1)\right\}\right) \\
& t^{j i}(k)=\arg \min _{t^{j i}} \mathcal{L}^{c}\left(\left\{x^{j}(k-1)\right\},\left\{t^{j i}\right\} ;\left\{u^{j i}(k-1), v^{j i}(k-1)\right\}\right)
\end{aligned}
$$

or, since $\mathcal{L}^{c}$ is quadratic in $t^{i j}, t^{j i}$, in closed-form as

$$
\begin{aligned}
& t^{i j}(k)=\frac{u^{i j}(k-1)+v^{i j}(k-1)}{2 c}+\frac{x^{i}(k-1)+x^{j}(k-1)}{2} \\
& t^{j i}(k)=\frac{u^{j i}(k-1)+v^{j i}(k-1)}{2 c}+\frac{x^{j}(k-1)+x^{i}(k-1)}{2}
\end{aligned}
$$

For each player $i \in V$ we consider an augmented Lagrangian associated to (A.1), where $c>0$,

$$
\begin{aligned}
& \mathcal{L}_{i}^{c}\left(x^{i}, t^{i j}, t^{j i} ;\left\{u^{i j}, v^{j i}\right\}\right):=J_{i}\left(x_{i}^{i}, x_{-i}^{i}\right)+\mathcal{I}_{\Omega_{i}}\left(x_{i}^{i}\right) \\
& +\sum_{j \in N_{i}} u^{i j}\left(x^{i}-t^{i j}\right)+\sum_{j \in N_{i}} v^{j i^{T}}\left(x^{i}-t^{j i}\right) \\
& +\frac{c}{2} \sum_{j \in N_{i}}\left\|x^{i}-t^{i j}\right\|^{2}+\frac{c}{2} \sum_{j \in N_{i}}\left\|x^{i}-t^{j i}\right\|^{2}
\end{aligned}
$$

For player $i$ its dual variables $\left\{u^{i j}, v^{j i}\right\}$ are updated by dual ascent with $c$ as the step-size, based on (A.4),

$$
\begin{aligned}
& u^{i j}(k)=u^{i j}(k-1)+c\left(x^{i}(k-1)-t^{i j}(k)\right), \\
& v^{j i}(k)=v^{j i}(k-1)+c\left(x^{i}(k-1)-t^{j i}(k)\right) .
\end{aligned}
$$

Similarly, for player $j$ 's, based on $\mathcal{L}_{j}^{c}$, its $\left\{u^{j i}, v^{i j}\right\}$ are,

$$
\begin{aligned}
& u^{j i}(k)=u^{j i}(k-1)+c\left(x^{j}(k-1)-t^{j i}(k)\right), \\
& v^{i j}(k)=v^{i j}(k-1)+c\left(x^{j}(k-1)-t^{i j}(k)\right) .
\end{aligned}
$$

Considering $u^{i j}(0)=v^{i j}(0)=u^{j i}(0)=v^{j i}(0)=\mathbf{0}_{N}$ and using (A.3) in (A.5),(A.6) yields

$$
\begin{array}{ll}
u^{i j}(k)+v^{i j}(k)=\mathbf{0}_{N} & \forall k \geq 0 \\
u^{j i}(k)+v^{j i}(k)=\mathbf{0}_{N} & \forall k \geq 0 .
\end{array}
$$

so that (A.3) become,

$$
t^{i j}(k)=\frac{x^{i}(k-1)+x^{j}(k-1)}{2}=t^{j i}(k) .
$$

Using (A.7) to eliminate $t^{i j}(k), t^{j i}(k)$ from (A.5), yields

$$
\begin{aligned}
& u^{i j}(k)=u^{i j}(k-1)+\frac{c}{2}\left(x^{i}(k-1)-x^{j}(k-1)\right) \\
& v^{j i}(k)=v^{j i}(k-1)+\frac{c}{2}\left(x^{i}(k-1)-x^{j}(k-1)\right) .
\end{aligned}
$$

Then for $w^{i}(k):=\sum_{j \in N_{i}} u^{i j}(k)+v^{j i}(k)$, this yields

$$
w^{i}(k)=w^{i}(k-1)+c \sum_{j \in N_{i}}\left(x^{i}(k-1)-x^{j}(k-1)\right)
$$

where $c>0$, as in Step 5 of Algorithm 1. Thus, the auxiliary variables $t^{i j}, t^{j i}$ can be eliminated using (A.7) and, based on (A.4), for each player $i \in V$ only the dual variables $w^{i}(k)$ (A.8) are needed to update his action $x_{i}^{i}(k)$ and estimates $x_{-i}^{i}(k)$, using his and his neighbours' previous estimates, $x^{i}(k-1), x^{j}(k-1), j \in N_{i}$. Specifically, minimizing (A.4) w.r.t. $x_{i}^{i}$ and with (A.7), for each $i \in V$ the local update for $x_{i}^{i}(k)=x_{i}(k)$ (decision) is

$$
\begin{aligned}
& x_{i}^{i}(k)=\arg \min _{x_{i}^{i} \in \mathbb{R}}\left\{J_{i}\left(x_{i}^{i}, x_{-i}^{i}(k-1)\right)+\mathcal{I}_{\Omega_{i}}\left(x_{i}^{i}\right)\right. \\
& \left.+w_{i}^{i}(k)^{T} x_{i}^{i}+c \sum_{j \in N_{i}}\left\|x_{i}^{i}-\frac{x_{i}^{i}(k-1)+x_{i}^{j}(k-1)}{2}\right\|^{2}\right\} .
\end{aligned}
$$

Note that in DOP, where the cost $f_{i}\left(x_{i}\right)$ is separable in $x_{i}$ (cf. (1)), in the update of $x_{i}$, coupling appears only linearly due to the quadratic terms, e.g., Wei and Ozdaglar (2013), Hong et al. (2016). In contrast, in (A.9) the $x_{i}^{2}$ problem is coupled nonlinearly to $x_{-i}^{i}(k-1)$ (due to the inherent coupling in $\left.J_{i}\right)$ and linearly to $x_{i}^{j}(k-1)$. As in an inexact $A D M M$, instead of solving exactly an optimization sub-problem, each player uses a linear proximal approximation to reduce the complexity of each subproblem. We approximate $J_{i}\left(x_{i}^{i}, x_{-i}^{i}(k-1)\right)$ in $(\mathrm{A} .9)$ by a proximal first-order approximation around $x_{i}^{i}(k-1)$, but inspired by Hong et al. (2016), with an extra quadratic penalty, which yields

$$
\begin{aligned}
x_{i}^{i}(k)= & \arg \min _{x_{i}^{i} \in \mathbb{R}}\left\{\nabla_{i} J_{i}\left(x^{i}(k-1)\right)^{T}\left(x_{i}^{i}-x_{i}^{i}(k-1)\right)\right. \\
& +\frac{\beta_{i}}{2}\left\|x_{i}^{i}-x_{i}^{i}(k-1)\right\|^{2}+\mathcal{I}_{\Omega_{i}}\left(x_{i}^{i}\right)
\end{aligned}
$$




$$
\left.+w_{i}^{i}(k)^{T} x_{i}^{i}+\bar{c} \sum_{j \in N_{i}}\left\|x_{i}^{i}-\frac{x_{i}^{i}(k-1)+x_{i}^{j}(k-1)}{2}\right\|^{2}\right\},
$$

where $\beta_{i}>0, \bar{c}=c+c_{0}, c_{0} \geq 0$. This is equivalent to

$$
\begin{array}{r}
x_{i}^{i}(k)=\arg \min _{x_{i}^{i} \in \mathbb{R}}\left\{\mathcal{I}_{\Omega_{i}}\left(x_{i}^{i}\right)+\frac{\alpha_{i}}{2} \| x_{i}^{i}-\alpha_{i}^{-1}\left[-\nabla_{i} J_{i}\left(x^{i}(k-1)\right)\right.\right. \\
\left.\left.+\beta_{i} x_{i}^{i}(k-1)-w_{i}^{i}(k)+\bar{c} \sum_{j \in N_{i}}\left(x_{i}^{i}(k-1)+x_{i}^{j}(k-1)\right)\right] \|^{2}\right\},
\end{array}
$$

where $\alpha_{i}=\beta_{i}+2 \bar{c}\left|N_{i}\right|$. Using (5) and $\operatorname{prox}_{\mathcal{I}_{\Omega_{i}}}^{\alpha_{i}}\{\cdot\}=$ $T_{\Omega_{i}}\{\cdot\}$, each player $i$ updates its action $x_{i}^{i}(k)=x_{i}(k)$ as in Step 6 of Algorithm 1. Finally, each player $i$ updates its estimates of the other players' actions $x_{-i}^{i}(k)$. This update is similar to (A.9) but ignoring the $J_{i}\left(x_{i}^{i}, x_{-i}^{i}\right)$ term (which is minimized only w.r.t. $x_{i}^{i}$, see (A.1)), i.e.,

$$
\begin{aligned}
& x_{-i}^{i}(k)=\arg \min _{x_{-i}^{i} \in \mathbb{R}^{N-1}}\left\{w_{-i}^{i}(k)^{T} x_{-i}^{i}\right. \\
& \left.\quad+\bar{c} \sum_{j \in N_{i}}\left\|x_{-i}^{i}-\frac{x_{-i}^{i}(k-1)+x_{-i}^{j}(k-1)}{2}\right\|^{2}\right\} .
\end{aligned}
$$

Following the same proximal approximation, the update for $x_{-i}^{i}(k)$ is obtained as in Step 7 of Algorithm 1.

\section{B Proof of Proposition 1}

The stacked vector update for $\mathbf{w},(18)$, follows from Step 5 of Algorithm 1. From Step 6 in Algorithm 1, $\alpha_{i} x_{i}^{i}(k)=T_{\Omega_{i}}\{y\}=\operatorname{prox}_{\mathcal{I}_{\Omega_{i}}}\{y\}$, where $y$ is the term in the square-bracket on the right-hand side of Step 6 . From Proposition 16.34, Bauschke and Combettes

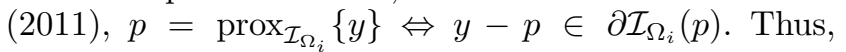
$\alpha_{i} x_{i}^{i}(k)=\operatorname{prox}_{\mathcal{I}_{\Omega_{i}}}\{y\} \Leftrightarrow y-\alpha_{i} x_{i}^{i}(k) \in \partial \mathcal{I}_{\Omega_{i}}\left(\alpha_{i} x_{i}^{i}(k)\right)$ $\Leftrightarrow y-\alpha_{i} x_{i}^{i}(k) \in \partial \mathcal{I}_{\Omega_{i}}\left(x_{i}^{i}(k)\right)$ since $\alpha_{i}>0$. Expanding $y$ as on the right-hand side of Step 6 and using $\alpha_{i}=\beta_{i}+2 \bar{c}\left|N_{i}\right|>0$ this is equivalent to

$\mathbf{0} \in \nabla_{i} J_{i}\left(x_{i}^{i}(k-1), x_{-i}^{i}(k-1)\right)+\partial_{i} \mathcal{I}_{\Omega_{i}}\left(x_{i}^{i}(k)\right)+w_{i}^{i}(k)+$ $\left(\beta_{i}+2 \bar{c}\left|N_{i}\right|\right) x_{i}^{i}(k)-\beta_{i} x_{i}^{i}(k-1)-\bar{c} \sum_{j \in N_{i}}\left(x_{i}^{i}(k-1)+x_{i}^{j}(k-1)\right)$

i.e.,

$\mathbf{0} \in \nabla_{i} J_{i}\left(x_{i}^{i}(k-1), x_{-i}^{i}(k-1)\right)+\partial_{i} \mathcal{I}_{\Omega_{i}}\left(x_{i}^{i}(k)\right)+w_{i}^{i}(k)+$ $\left(\beta_{i}+2 \bar{c} \mid N_{i}\right)\left(x_{i}^{i}(k)-x_{i}^{i}(k-1)\right)+\bar{c} \sum_{j \in N_{i}}\left(x_{i}^{i}(k-1)-x_{i}^{j}(k-1)\right)$.

From Step 7 in Algorithm 1, one can obtain for $x_{-i}^{i}$,

$$
\begin{aligned}
\mathbf{0}_{N-1} & =\left(\beta_{i}+2 \bar{c}\left|N_{i}\right|\right)\left(x_{-i}^{i}(k)-x_{-i}^{i}(k-1)\right)+w_{-i}^{i}(k) \\
& +\bar{c} \sum_{j \in N_{i}}\left(x_{-i}^{i}(k-1)-x_{-i}^{j}(k-1)\right) .
\end{aligned}
$$

Combining these two relations into a single vector one for all components of $x^{i}=\left(x_{i}^{i}, x_{-i}^{i}\right)$, yields $\forall i \in V$

$$
\begin{gathered}
\mathbf{0}_{N} \in \nabla_{i} J_{i}\left(x^{i}(k-1)\right) e_{i}+\partial_{i} \mathcal{I}_{\Omega_{i}}\left(x_{i}^{i}(k)\right) e_{i}+w^{i}(k)+ \\
\left(\beta_{i}+2 \bar{c} \mid N_{i}\right)\left(x_{i}^{i}(k)-x_{i}^{i}(k-1)\right)+\bar{c} \sum_{j \in N_{i}}\left(x_{i}^{i}(k-1)-x_{i}^{j}(k-1)\right) .
\end{gathered}
$$

In stacked vector form for all $i \in V$ this yields

$$
\begin{aligned}
\mathbf{0}_{N^{2}} & \in\left[\nabla_{i} J_{i}\left(x^{i}(k-1)\right) e_{i}\right]_{i \in V}+\left[\partial_{i} \mathcal{I}_{\Omega_{i}}\left(x_{i}^{i}(k)\right) e_{i}\right]_{i \in V}+\mathbf{w}(k) \\
& +\left((\mathbb{B}+2 \bar{c} \mathbb{D}) \otimes I_{N}\right)(\mathbf{x}(k)-\mathbf{x}(k-1)) \\
& +\bar{c}\left(L \otimes I_{N}\right) \mathbf{x}(k-1),
\end{aligned}
$$

where $\mathbf{x}=\left[x^{i}\right]_{i \in V}, \mathbf{w}=\left[w^{i}\right]_{i \in V}, \mathbb{B}=\operatorname{diag}\left(\left[\beta_{i}\right]_{i \in V}\right)$, $\mathbb{D}=\operatorname{diag}\left(\left[\left|N_{i}\right|\right]_{i \in V}\right)$, which can be written as $(18)$.

\section{Proof of Lemma 2}

We decompose the augmented space $\mathbb{R}^{N^{2}}$ into the consensus subspace $\operatorname{Ker}(\mathbf{L})=\left\{\mathbf{1}_{N} \otimes x \mid x \in \mathbb{R}^{N}\right\}$ and its orthogonal complement $\operatorname{Ker}(\mathbf{L})^{\perp}$. Any $\mathbf{x} \in \mathbb{R}^{N^{2}}$ can be decomposed as $\mathbf{x}=\mathbf{x}^{\|}+\mathbf{x}^{\perp}$, with $\mathbf{x}^{\|} \in \operatorname{Ker}(\mathbf{L}), \mathbf{x}^{\perp} \in$ $\operatorname{Ker}(\mathbf{L})^{\perp},\left(\mathbf{x}^{\|}\right)^{T} \mathbf{x}^{\perp}=0$, Thus $\mathbf{x}^{\|}=\mathbf{1}_{N} \otimes x$, for some $x \in$

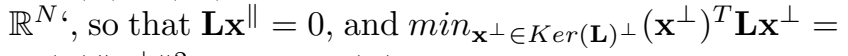
$\lambda_{2}(L)\left\|\mathbf{x}^{\perp}\right\|^{2}$, where $\lambda_{2}(L)>0$. Similarly, we can write any $\mathbf{y} \in \operatorname{Ker}(\mathbf{L})$ as $\mathbf{y}=\mathbf{1}_{N} \otimes y$, for some $y \in \mathbb{R}^{N}$ and $\mathbf{L y}=0$. Then, using $\mathbf{x}=\mathbf{x}^{\|}+\mathbf{x}^{\perp}, \mathbf{F}\left(\mathbf{x}^{\|}\right)=F(x)$, $\mathbf{F}(\mathbf{y})=F(y), \mathbf{R}^{T} \mathbf{x}^{\|}=x, \mathbf{R}^{T} \mathbf{y}=y$ we can write

$$
\begin{aligned}
&(\mathbf{x}-\mathbf{y})^{T}\left(\mathbf{R}(\mathbf{F}(\mathbf{x})-\mathbf{F}(\mathbf{y}))+c_{0} \mathbf{L}(\mathbf{x}-\mathbf{y})\right) \\
&=\left(\mathbf{x}^{\|}-\mathbf{y}\right)^{T} \mathbf{R}\left(\mathbf{F}(\mathbf{x})-\mathbf{F}\left(\mathbf{x}^{\|}\right)+\mathbf{F}\left(\mathbf{x}^{\|}\right)-\mathbf{F}(\mathbf{y})\right) \\
& \quad+\left(\mathbf{x}^{\perp}\right)^{T} \mathbf{R}\left(\mathbf{F}(\mathbf{x})-\mathbf{F}\left(\mathbf{x}^{\|}\right)+\mathbf{F}\left(\mathbf{x}^{\|}\right)-\mathbf{F}(\mathbf{y})\right) \\
&\left.\quad+c_{0}\left(\mathbf{x}^{\|}+\mathbf{x}^{\perp}-\mathbf{y}\right)^{T} \mathbf{L}\left(\mathbf{x}^{\|}+\mathbf{x}^{\perp}-\mathbf{y}\right)\right) \\
&=(x-y)^{T}\left(\mathbf{F}(\mathbf{x})-\mathbf{F}\left(\mathbf{x}^{\|}\right)\right)+(x-y)^{T}(F(x)-F(y)) \\
&+\left(\mathbf{x}^{\perp}\right)^{T} \mathbf{R}\left(\mathbf{F}(\mathbf{x})-\mathbf{F}\left(\mathbf{x}^{\|}\right)\right)+\left(\mathbf{x}^{\perp}\right)^{T} \mathbf{R}(F(x)-F(y)) \\
&+c_{0}\left(\mathbf{x}^{\perp}\right)^{T} \mathbf{L} \mathbf{x}^{\perp}
\end{aligned}
$$

Using strong monotonicity of $F$ (by Assumption 2) for the second term, and properties of $\mathbf{L}$ on $\operatorname{Ker}(\mathbf{L})^{\perp}$ for the fifth term on the right-hand side we can write,

$$
\begin{aligned}
& (\mathbf{x}-\mathbf{y})^{T}\left(\mathbf{R}(\mathbf{F}(\mathbf{x})-\mathbf{F}(\mathbf{y}))+c_{0} \mathbf{L}(\mathbf{x}-\mathbf{y})\right) \\
& \geq(x-y)^{T}\left(\mathbf{F}(\mathbf{x})-\mathbf{F}\left(\mathbf{x}^{\|}\right)\right)+\mu\|x-y\|^{2} \\
& \quad+\left(\mathbf{x}^{\perp}\right)^{T} \mathbf{R}\left(\mathbf{F}(\mathbf{x})-\mathbf{F}\left(\mathbf{x}^{\|}\right)\right)+\left(\mathbf{x}^{\perp}\right)^{T} \mathbf{R}(F(x)-F(y)) \\
& \quad+c_{0} \lambda_{2}(L)\left\|\mathbf{x}^{\perp}\right\|^{2}
\end{aligned}
$$

We deal with the cross-terms by using $a^{T} b \geq-\|a\|\|b\|$, for any $a, b$, and $\|\mathbf{R} b\| \leq\|\mathbf{R}\|_{2}\|b\|$, so that

$$
\begin{aligned}
& (\mathbf{x}-\mathbf{y})^{T}\left(\mathbf{R}(\mathbf{F}(\mathbf{x})-\mathbf{F}(\mathbf{y}))+c_{0} \mathbf{L}(\mathbf{x}-\mathbf{y})\right) \geq \\
& \quad-\|x-y\|\left\|\mathbf{F}(\mathbf{x})-\mathbf{F}\left(\mathbf{x}^{\|}\right)\right\|+\mu\|x-y\|^{2} \\
& \quad-\left\|\mathbf{x}^{\perp}\right\|\|\mathbf{R}\|_{2}\left\|\mathbf{F}(\mathbf{x})-\mathbf{F}\left(\mathbf{x}^{\|}\right)\right\|-\left\|\mathbf{x}^{\perp}\right\|\|\mathbf{R}\|_{2}\|F(x)-F(y)\| \\
& \quad+c_{0} \lambda_{2}(L)\left\|\mathbf{x}^{\perp}\right\|^{2}
\end{aligned}
$$

Using $\|\mathbf{R}\|_{2}=1$ and Lipschitz properties of $F$ and $\mathbf{F}$,

$$
\begin{aligned}
& (\mathbf{x}-\mathbf{y})^{T}\left(\mathbf{R}(\mathbf{F}(\mathbf{x})-\mathbf{F}(\mathbf{y}))+c_{0} \mathbf{L}(\mathbf{x}-\mathbf{y})\right) \geq \\
& \quad-\theta\|x-y\|\left\|\mathbf{x}^{\perp}\right\|+\mu\|x-y\|^{2}-\theta\left\|\mathbf{x}^{\perp}\right\|^{2}-\theta_{0}\left\|\mathbf{x}^{\perp}\right\|\|x-y\|
\end{aligned}
$$




$$
+c_{0} \lambda_{2}(L)\left\|\mathbf{x}^{\perp}\right\|^{2}
$$

Using $\|x-y\|=\frac{1}{\sqrt{N}}\left\|\mathbf{x}^{\|}-\mathbf{y}\right\|$ and $\Psi,(24)$, we can write

$(\mathbf{x}-\mathbf{y})^{T}\left(\mathbf{R}(\mathbf{F}(\mathbf{x})-\mathbf{F}(\mathbf{y}))+c_{0} \mathbf{L}(\mathbf{x}-\mathbf{y})\right) \geq\left[\begin{array}{c}\left\|\mathbf{x}^{\|}-\mathbf{y}\right\| \\ \left\|\mathbf{x}^{\perp}\right\|\end{array}\right]^{T} \Psi\left[\begin{array}{c}\left\|\mathbf{x}^{\|}-\mathbf{y}\right\| \\ \left\|\mathbf{x}^{\perp}\right\|\end{array}\right]$

Thus,

$(\mathbf{x}-\mathbf{y})^{T}\left(\mathbf{R}(\mathbf{F}(\mathbf{x})-\mathbf{F}(\mathbf{y}))+c_{0} \mathbf{L}(\mathbf{x}-\mathbf{y})\right) \geq \bar{\mu}\left(\left\|\mathbf{x}^{\|}-\mathbf{y}\right\|^{2}+\left\|\mathbf{x}^{\perp}\right\|^{2}\right)$,

where $\bar{\mu}:=\lambda_{\min }(\Psi)$. Using $\|\mathbf{x}\|-\mathbf{y}\left\|^{2}+\right\| \mathbf{x}^{\perp}\left\|^{2}=\right\| \mathbf{x}-\mathbf{y} \|^{2}$, this can be written as (25). For $c_{0}$ as in the statement, $\Psi \succ 0$, hence $\bar{\mu}>0$.

\section{References}

Agarwal, A., Negahban, S. and Wainwright, M. J. (2010), Fast global convergence rates of gradient methods for high-dimensional statistical recovery, in 'Advances in Neural Information Processing Systems 23', pp. 37-45.

Alpcan, T. and Basar, T. (2002), A game-theoretic framework for congestion control in general topology networks, in 'Proc. 41st IEEE Conference on Decision and Control (CDC)', Vol. 2, pp. 1218-1224.

Alpcan, T. and Başar, T. (2005), Distributed algorithms for Nash equilibria of flow control games, in 'Advances in Dynamic Games', Springer, pp. 473-498.

Bauschke, H. and Combettes, P. (2011), Convex analysis and monotone operator theory in Hilbert spaces, Springer Science \& Business.

Bertsekas, D. P. and Tsitsiklis, J. N. (1997), Parallel and Distributed Computation: Numerical Methods, Athena Scientific, Belmont, Massachusetts.

Bimpikis, K., Ehsani, S. and Ilkilic, R. (2014), Cournot competition in networked markets, in 'Proc. 15th ACM conference on Economics and computation', p. 733 .

Bimpikis, K., Ozdaglar, A. and Yildiz, E. (2016), 'Competitive Targeted Advertising over Networks', Operations Research 64(3), 705-720.

Boyd, S., Parikh, N., Chu, E., Peleato, B. and Eckstein, J. (2011), 'Distributed optimization and statistical learning via the alternating direction method of multipliers', Foundations and Trends® in Machine Learning 3(1), 1-122.

Bramoullé, Y., Kranton, R. and D'amours, M. (2014), 'Strategic interaction and networks', The American Economic Review 104(3), 898-930.

Chang, T.-H., Hong, M. and Wang, X. (2015), 'Multiagent distributed optimization via inexact consensus ADMM', IEEE Transactions on Signal Processing 63(2), 482-497.

Cheng, N., Zhang, N., Lu, N., Shen, X., Mark, J. W. and Liu, F. (2014), 'Opportunistic Spectrum Access for
CR-VANETs: A Game-Theoretic Approach', IEEE Trans. in Vehicular Technology 63(1), 237-250.

Chung, F. R. (1997), Spectral graph theory, Vol. 92, American Mathematical Soc.

Debreu, G. (1952), 'A Social Equilibrium Existence Theorem', Proceedings of the National Academy of Sciences of the United States of America 38(10), 886.

Facchinei, F. and Pang, J.-S. (2007), Finite-dimensional variational inequalities and complementarity problems, Vol. I and I, Springer.

Frihauf, P., Krstic, M. and Basar, T. (2012), 'Nash equilibrium seeking in noncooperative games', IEEE Transactions on Automatic Control 57(5), 1192-1207.

Gadjov, D. and Pavel, L. (2018), 'A Passivity-Based Approach to Nash Equilibrium Seeking over Networks', IEEE Transactions on Automatic Control (Early Access) 1-1(DOI: 10.1109/TAC.2018.2833140).

Ghaderi, J. and Srikant, R. (2014), 'Opinion dynamics in social networks with stubborn agents: Equilibrium and convergence rate', Automatica 50(2), 3209-3215.

Glicksberg, I. (1952), 'A Further Generalization of the Kakutani Fixed Point Theorem, with Application to Nash Equilibrium Points', Proceedings of the American Mathematical Society 3(1), 170-174.

Hong, M., Luo, Z.-Q. and Razaviyayn, M. (2016), 'Convergence analysis of alternating direction method of multipliers for a family of nonconvex problems', SIAM Journal on Optimization 26(1), 337-364.

Kannan, A. and Shanbhag, U. (2012), 'Distributed computation of equilibria in monotone Nash games via iterative regularization techniques', SIAM Journal on Optimization 22(4), 1177-1205.

Koshal, J., Nedic, A. and Shanbhag, U. V. (2016), 'Distributed algorithms for aggregative games on graphs', Operations Research 64(3), 680-704.

Li, N. and Marden, J. R. (2013), 'Designing games for distributed optimization', IEEE Journal of Selected Topics in Signal Processing 7(2), 230-242.

Li, S. and Başar, T. (1987), 'Distributed Algorithms for the Computation of Noncooperative Equilibria', $A u$ tomatica 23(4), 523-533.

Marden, J. (2007), 'Learning in large-scale games and cooperative control', Ph.D. Dissertation .

Nash, J. (1950), 'Equilibrium Points in $n$-Person Games', Proc. Nat. Acad. Sci. USA 36(1), 48-49.

Negahban, S., Yu, B., Wainwright, M. J. and Ravikumar, P. K. (2009), A unified framework for highdimensional analysis of M-estimators with decomposable regularizers, in 'Advances in Neural Information Processing Systems 22', pp. 1348-1356.

Pan, Y. and Pavel, L. (2009), 'Games with coupled propagated constraints in optical networks with multi-link topologies', Automatica 45(4), 871-880.

Parise, F., Gentile, B., Grammatico, S. and Lygeros, J. (2015), Network aggregative games: Distributed convergence to Nash equilibria, in 'Proc. 54th IEEE Conference on Decision and Control (CDC)', pp. 22952300.

Poliquin, R. and Rockafellar, R. (1996), 'Prox-regular 
functions in variational analysis', Transactions of the American Mathematical Society 348(5), 1805-1838.

Rockafellar, R. (1970), 'On the Maximality of Sums of Nonlinear Monotone Operators', Transactions of the American Mathematical Society 149(1), 75-88.

Salehisadaghiani, F. and Pavel, L. (2016), 'Distributed Nash equilibrium seeking: A gossip-based algorithm', Automatica 72, 209-216.

Salehisadaghiani, F. and Pavel, L. (2017), 'Distributed Nash equilibrium seeking via the alternating direction method of multipliers', IFAC-PapersOnLine 50(1), 6166-6171. 20th IFAC World Congress.

Salehisadaghiani, F. and Pavel, L. (2018), 'Distributed Nash equilibrium seeking in networked graphical games', Automatica 87, 17-24.

Scutari, G., Facchinei, F., Pang, J. S. and Palomar, D. P. (2014), 'Real and Complex Monotone Communication Games', IEEE Trans. on Information Theory 60(7), 4197-4231.

Shi, W., Ling, Q., Wu, G. and Yin, W. (2015), 'A proximal gradient algorithm for decentralized composite optimization', IEEE Transactions on Signal Processing 63(22), 6013-6023.

Shi, W., Ling, Q., Yuan, K., Wu, G. and Yin, W. (2014), 'On the linear convergence of the ADMM in decentralized consensus optimization', IEEE Transactions on Signal Processing 62(7), 1750-1761.

Shi, W. and Pavel, L. (2017), LANA: an ADMM-like Nash equilibrium seeking algorithm in decentralized environment, in 'Proc. American Control Conference', pp. 285-290.

Stanković, M., Johansson, K. and Stipanović, D. (2012), 'Distributed Seeking of Nash Equilibria with Applications to Mobile Sensor Networks', IEEE Transactions on Automatic Control 57(4), 904-919.

Swenson, B., Kar, S. and Xavier, J. (2015), 'Empirical centroid fictitious play: An approach for distributed learning in multi-agent games', Signal Processing, IEEE Transactions on 63(15), 3888-3901.

Tekin, C., Liu, M., Southwell, R., Huang, J. and Ahmad, S. (2012), 'Atomic congestion games on graphs and their applications in networking', IEEE/ACM Trans. on Networking 20(5), 1541-1552.

Wang, B., Wu, Y. and Ray Liu, K. J. (2010), 'Game theory for cognitive radio networks: An overview', Computer Networks 54, 2537-2561.

Wang, Y., Yin, W. and Zeng, J. (2015), 'Global convergence of ADMM in nonconvex nonsmooth optimization', arXiv preprint arXiv:1511.06324v6 .

Wei, E. and Ozdaglar, A. (2012), Distributed alternating direction method of multipliers, in 'Proc. 51st IEEE Conference on Decision and Control (CDC)', pp. 5445-5450.

Wei, E. and Ozdaglar, A. (2013), 'On the o(1/k) convergence of asynchronous distributed alternating direction method of multipliers', arXiv preprint arXiv:1307.8254v1.

Ye, M. and Hu, G. (2017), 'Distributed Nash Equilibrium Seeking by A Consensus Based Approach', IEEE
Transactions on Automatic Control 62(9), 4811-4818. Yi, P. and Pavel, L. (2017), A distributed primal-dual algorithm for computation of generalized Nash equilibria via operator splitting methods, in 'Proc. 56th IEEE Conference on Decision and Control (CDC)', pp. 3841-3846.

Yi, P. and Pavel, L. (2018), 'Distributed generalized Nash equilibria computation of monotone games via double-layer preconditioned proximal-point algorithms', IEEE Transactions on Control of Network Systems $\mathbf{P P}(99), 1-1$.

Yin, H., Shanbhag, U. V. and Mehta, P. G. (2011), 'Nash equilibrium problems with scaled congestion costs and shared constraints', IEEE Transactions on Automatic Control 56(7), 1702-1708.

Yousefian, F., Nedich, A. and Shanbhag, U. V. (2016), 'Self-Tuned Stochastic Approximation Schemes for Non-Lipschitzian Stochastic Multi-User Optimization and Nash Games', IEEE Transactions on Automatic Control 61(7), 1753-1766.

Zhang, H. (2017), 'The restricted strong convexity revisited: analysis of equivalence to error bound and quadratic growth', Optimization Lett. 11(4), 817-833.

Zhu, M. and Frazzoli, E. (2016), 'Distributed robust adaptive equilibrium computation for generalized convex games', Automatica 63, 82-91. 